Estudo de simetria e seu ensino no nível fundamental e médio

Elisandra Regina Sampaio dos Reis 


\title{
Estudo de simetria e seu ensino no nível fundamental e médio
}

\author{
Elisandra Regina Sampaio dos Reis
}

Orientador: Prof. Dr. Oziride Manzoli Neto

Dissertação apresentada ao Instituto de Ciências Matemáticas e de Computação - ICMC-USP, como parte dos requisitos para obtenção do título de Mestre Programa de Mestrado Profissional em Matemática. VERSÃO REVISADA 
Ficha catalográfica elaborada pela Biblioteca Prof. Achille Bassi e Seção Técnica de Informática, ICMC/USP, com os dados fornecidos pelo(a) autor(a)

\begin{tabular}{|c|c|}
\hline \multirow[t]{3}{*}{ R375e } & $\begin{array}{l}\text { Reis, Elisandra Regina Sampaio dos } \\
\text { Estudo de simetria e seu ensino no nível } \\
\text { fundamental e médio / Elisandra Regina Sampaio dos } \\
\text { Reis; orientador Oziride Manzoli Neto. -- São } \\
\text { Carlos, } 2013 \text {. } \\
\quad 52 \mathrm{p} \text {. }\end{array}$ \\
\hline & $\begin{array}{l}\text { Dissertação (Mestrado - Programa de Pós-Graduação } \\
\text { em Mestrado Profissional em Matemática em Rede } \\
\text { Nacional) -- Instituto de Ciências Matemáticas e de } \\
\text { Computação, Universidade de São Paulo, 2013. }\end{array}$ \\
\hline & $\begin{array}{l}\text { 1. simetria. 2. grupos. 3. transformações } \\
\text { geométricas. I. Manzoli Neto, Oziride, orient. II. } \\
\text { Título. }\end{array}$ \\
\hline
\end{tabular}


AUtORIZO A REPRODUÇÃo E DiVULGAÇÃo TOTAL OU PARCIAL DESTE TRABALHO, POR QUALQUER MEIO CONVENCIONAL OU ELETRÔNICO, PARA Fins DE ESTUdo E PESQUisa, DESDE QUE CitAda A FOnTE. 


\title{
RESUMO
}

Reis, E. R. S. (2013). Estudo de Simetria e seu ensino no nível fundamental e médio. Dissertação de Mestrado, Instituto de Ciências Matemáticas e de Computação. Universidade de São Paulo, São Carlos.

Esse trabalho tem por objetivo estudar Teoria dos Grupos focando nos Grupos de Simetrias, destacar a importância desse estudo e analisar estratégias para ensinar o conceito de simetria de forma inteligível para os alunos do ensino fundamental II e ensino médio.

Palavras-chave: Simetria, Grupos.

\begin{abstract}
Reis, E. R. S. (2013).Study of Symmetry and its teaching in elementary and high school. MSc Thesis, Institute of Mathematics and Computer. University of São Paulo, São Carlos.

The pourpose of this work is to study Group Theory focusing Groups of Symmetries, to reiterate the importance of this study and launch strategies for teaching the concept of simetry in an intelligible form for elementary and high school students.
\end{abstract}

Keywords: Symmetry, Groups. 


\section{SUMÁRIO}

Pagina

1 Introdução 2

2 Conceitos Geométricos Fundamentais 3

2.1 Figuras Geométricas . . . . . . . . . . . . . . . 3

2.1.1 Figuras Planas . . . . . . . . . . . . . . 3

2.1.2 Figuras Espaciais . . . . . . . . . . . . . . . . . . . . . . 4

2.2 Transformações Geométricas . . . . . . . . . . . . . . . . . . 4

2.2.1 Homotetias . . . . . . . . . . . . . . 4

2.2 .2 Isometrias . . . . . . . . . . . . . . . . . 5

2.2.3 Translações . . . . . . . . . . . . . . . . . . . 6

2.2.4 Rotações . . . . . . . . . . . . . . . . . 6

2.2 .5 Reflexões ..................... 11

3 Conceitos Algébricos Fundamentais $\quad 16$

3.1 Grupos . . . . . . . . . . . . . . . . 16

3.1.1 Grupo de Matrizes (com a operação multiplicação) . . 17

3.1.2 Grupo de Permutações . . . . . . . . . . . . . . . . . 18

3.1.3 Homomorfismo de Grupos . . . . . . . . . . . . . . . 18

3.1.4 Isomorfismo de Grupos . . . . . . . . . . . . . . . . . . 19

3.1.5 Grupos Isomorfos . . . . . . . . . . . . . . . . . 19

4 Grupos de Simetrias de Figuras Planas 20

4.1 Grupo de Simetrias de um Triângulo Isósceles . . . . . . . . . 20

4.2 Grupo de Simetrias de um Retângulo . . . . . . . . . . . . . . 21

4.3 Grupo de Simetrias de um Triângulo Equilátero . . . . . . . . 22

4.4 Grupo de Simetrias do Quadrado . . . . . . . . . . . . . . 23

4.5 Grupo de Simetrias do Pentágono Regular . . . . . . . . . . . 24

4.6 Grupo de Simetrias de um Polígono Regular de $n$ lados . . . . 26

5 Grupo de Simetrias de Poliedros 28

5.1 Grupo de Simetrias dos Prismas . . . . . . . . . . . . . . . . . 28

5.1.1 Grupo de Simetrias do Paralelepípedo . . . . . . . . 28

5.1.2 Grupo de Simetrias do Prisma Pentagonal Reto . . . . 30

5.1.3 Grupo de Simetrias de um Prisma de $n$ faces laterais . 33

5.2 Grupo de Simetrias das Pirâmides . . . . . . . . . . . . . . 33

5.2.1 Grupo de Simetrias da Pirâmide Hexagonal Reta . . . 33 
5.2.2 Grupo de Simetrias da Pirâmide Reta de $n$ faces laterais 35

5.3 Grupo de Simetrias dos Poliedros Regulares . . . . . . . . . . 36

5.3.1 Grupo de Simetrias do Tetraedro Regular . . . . . . . 37

5.3.2 Grupo de Simetrias do Hexaedro Regular ou Cubo . . 39

5.3.3 Grupo de Simetrias do Octaedro Regular . . . . . . . 40

5.3.4 Grupo de Simetrias do Dodecaedro Regular . . . . . . 41

5.3.5 Grupo de Simetrias do Icosaedro Regular . . . . . . . 42

6 Aplicações 43

7 Atividades $\quad 45$ 


\section{Introdução}

Muitas vezes ao observar a natureza vemos uma certa harmonia entre as formas e cores como, por exemplo, na maioria das borboletas a asa direita é igual a asa esquerda. E assim ao estabelecermos, mesmo que intuitivamente, uma correspondência entre as distâncias dos pontos que formam os desenhos das asas, estamos usando o conceito de um dos tipos de simetria.

Há registros de que os babilônios e os egípcios usavam o conceito de simetria em suas pinturas, artesanatos e para resolver problemas do cotidiano. Ao longo da história o homem utilizou esse conceito e eventualmente começou a formalizar a ideia usando linguagem matemática e com isso pôde compreender melhor o conceito de simetria e aperfeiçoar sua aplicação em várias áreas do conhecimento.

O estudo de simetria tornou-se importante e fundamental em diversas áreas do conhecimento como Arquitetura, Artes, Biologia, Física, Química, Matemática, Literatura entre outras.

No século XIX, Evariste Galois formalizou o conceito de Simetria Geométrica baseado na ideia de grupos de transformações. A ideia de grupo é usada para se obter uma estrutura no conjunto das transformações isométricas de um objeto em si mesmo.

Nosso objetivo é salientar a importância da formalização do conceito de simetria. Inicialmente focaremos nosso estudo na teoria de grupos (abstratos) dando como exemplos o conjunto das simetrias de alguns objetos geométricos mais simples como polígonos e poliedros, posteriormente citaremos exemplos de aplicações e daremos sugestões de como trabalhar essa formalização no ensino básico. 


\section{Conceitos Geométricos Fundamentais}

\subsection{Figuras Geométricas}

\section{Ponto e Reta}

Conceitos como ponto e reta são primitivos então os aceitaremos sem definição. O ponto possui "dimensão" zero e a reta possui "dimensão" um.

\subsubsection{Figuras Planas}

\section{Plano}

Um plano é um subconjunto do espaço $\mathbb{R}^{3}$ de tal modo que quaisquer dois pontos desse conjunto pode ser ligado por um segmento de reta inteiramente contido no conjunto. O plano posssui "dimensão" dois.

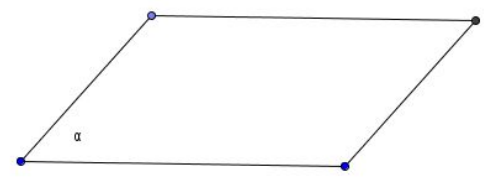

Figura 1:

\section{Polígono}

É uma figura plana e fechada delimitada por três ou mais segmentos de reta que se intersectam dois a dois apenas nos extremos. Os segmentos são denominados lados do polígono. Os pontos de intersecção são denominados vértices do polígono.

\section{Polígono Convexo}

É um polígono $P$ tal que se $A \in P$ e $B \in P$ então todo o segmento $A B$ está em $P$.

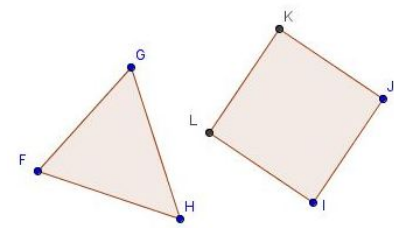

Figura 2: 


\subsubsection{Figuras Espaciais}

\section{Poliedros}

Poliedro é uma região espacial delimitada por quatro ou mais polígonos que se intersectam, dois a dois, apenas nos lados dos polígonos. Cada um desses polígonos chama-se face do poliedro, cada lado comum a duas faces chama-se aresta do poliedro e cada vértice de uma face é também chamado vértice do poliedro.

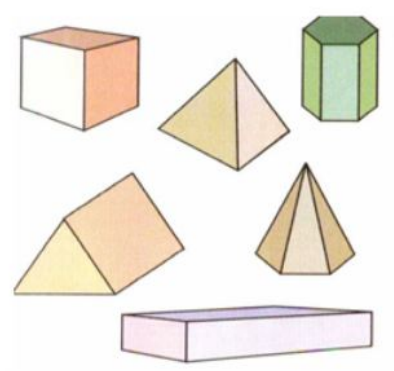

Figura 3:

\subsection{Transformações Geométricas}

Uma transformação geométrica é uma aplicação biunívoca $T: X \rightarrow Y$, onde X e Y são objetos geométricos.

Especificamente estudaremos transformações geométricas que levam uma figura a outra "geometricamente equivalente" e tomaremos em geral figuras nos espaços $\mathbb{R}, \mathbb{R}^{2}$ e $\mathbb{R}^{3}$, onde:

- o conjunto dos números reais $\mathbb{R}$ é representado geometricamente pela reta

- $\mathbb{R}^{2}=\mathbb{R} \times \mathbb{R}$ representado geometricamente pelo plano

- $\mathbb{R}^{3}=\mathbb{R}^{2} \times \mathbb{R}$ representado geometricamente pelo espaço.

\subsubsection{Homotetias}

Fixado um ponto $V$ do plano $\alpha$, e um número real $k>0$, a homotetia de centro $\mathrm{V}$ e razão $k$ é a transformação $H_{V, k}$, que associa a cada ponto $D$ do plano $\alpha$, o ponto $F=H_{V, k}(D)$ de $\alpha$, tal que a medida do segmento $V F$ é igual a $k$ vezes a medida de $V D(V F=k \cdot V D)$ e os pontos $V, D$ e $F$ são colineares. 
Uma homotetia preserva os ângulos, as razões entre os segmentos de reta e o paralelismo.

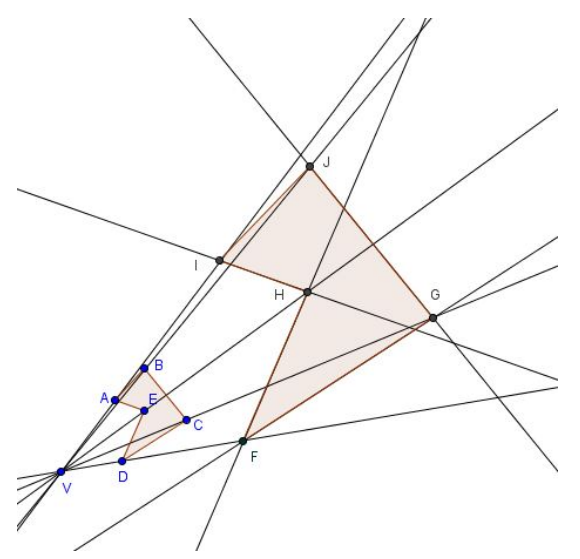

Figura 4:

\subsubsection{Isometrias}

Um espaço métrico $X$ é um conjunto munido de uma métrica $d: X \times X \rightarrow \mathbb{R}$ onde $d$ é chamada função distância que para quaisquer $x, y \mathrm{e}$ $z \in X$ satisfaz:

- $d(x, y) \geq 0$

- $d(x, y)=0 \Leftrightarrow x=y$

- $d(x, z) \leq d(x, y)+d(y, z)$

Uma aplicação $f: X \rightarrow Y$ sobrejetora entre espaços métricos é uma isometria se $d(f(x), f(y))=d(x, y)$ para todo $x, y \in X$, ou seja, isometria é uma transformação geométrica que, aplicada a uma figura geométrica, mantém as distâncias entre pontos, mantendo a amplitude dos ângulos e deixando a figura transformada "geometricamente equivalente" a figura inicial, podendo variar a direção e o sentido dos segmentos. As rotações, translações e reflexões são isometrias simples. Para uma melhor visualização do conceito considere a aplicação $f: \mathbb{R}^{2} \rightarrow \mathbb{R}^{2}$ tal que $f(A)=A^{\prime}$ e $f(B)=B^{\prime}$, conforme o desenho abaixo: 


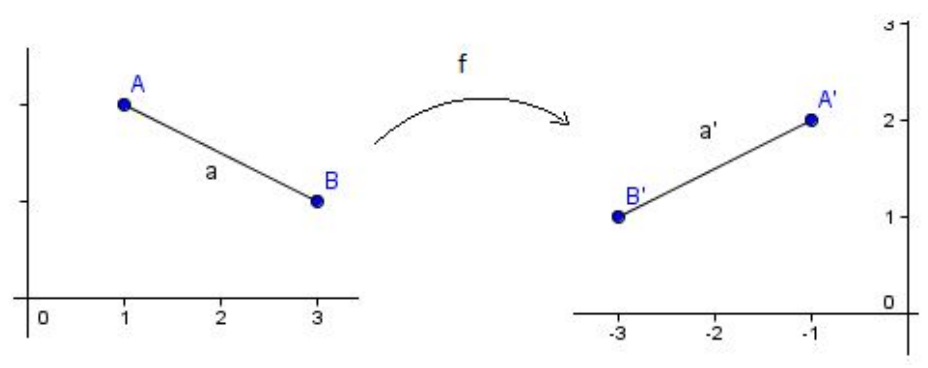

Figura 5:

\subsubsection{Translações}

No plano $\mathbb{R}^{2}$ uma translação $T: \mathbb{R}^{2} \rightarrow \mathbb{R}^{2}$ é definida por $T(x, y)=T(x+a, y+b)$, para fixados $a$ e $b \in \mathbb{R}$ e quaisquer $x, y \in \mathbb{R}$.

Seja $P\left(x_{1}, y_{1}\right)$ um ponto que é levado por uma translação, determinada por $(a, b)$ sobre uma reta $m$, a um ponto $Q\left(x_{2}, y_{2}\right)$ conforme mostra a figura abaixo:

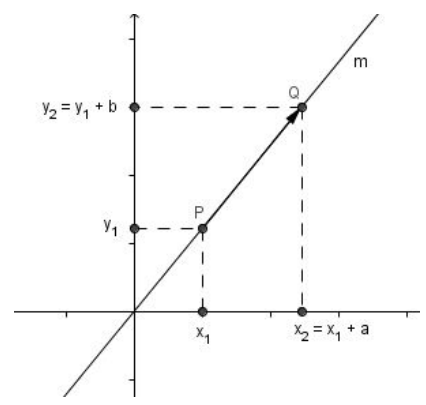

Figura 6:

Note que a translação desliza a figura na direção da reta $m$.

Então, de forma matricial para qualquer $\left(x_{1}, x_{2}\right) \in \mathbb{R}$, temos:

$$
T\left(\left[\begin{array}{l}
x_{1} \\
y_{1}
\end{array}\right]\right)=\left[\begin{array}{l}
x_{2} \\
y_{2}
\end{array}\right]=\left[\begin{array}{l}
x_{1} \\
y_{1}
\end{array}\right]+\left[\begin{array}{l}
a \\
b
\end{array}\right]=\left[\begin{array}{l}
x_{1}+a \\
x_{2}+b
\end{array}\right]
$$

\subsubsection{Rotações}

\section{Rotações em $\mathbb{R}^{2}$}

Rotação é um tipo de isometria que mantendo um ponto fixo $\mathrm{O}$ (centro de rotação) desloca cada ponto $P_{1}$ do objeto a um ponto $P_{2}$ sob um ângulo fixo $P_{1} \hat{O} P_{2}$ (denominado $\theta$ ). 
Seja $P_{1}\left(x_{1}, y_{1}\right)$ um ponto que é levado por uma rotação de centro na origem do sistema de coordenadas e ângulo $\theta$, no sentido anti-horário, ao ponto $P_{2}\left(x_{2}, y_{2}\right)$.

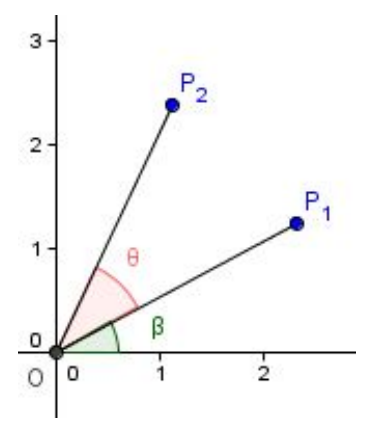

Figura 7:

Como a rotação é uma isometria então $d\left(O, P_{1}\right)=d\left(O, P_{2}\right)$ que consideremos igual a $r$ e assim teremos;

$$
\begin{aligned}
& x_{1}=r \cos \beta \\
& y_{1}=r \operatorname{sen} \beta \\
& \mathrm{e} \\
& x_{2}=r \cos (\beta+\theta)=r(\cos \beta \cdot \cos \theta-\operatorname{sen} \beta \cdot \operatorname{sen} \theta)=r \cos \beta \cdot \cos \theta-r \operatorname{sen} \beta \cdot \operatorname{sen} \theta \\
& x_{2}=x_{1} \cos \theta-y_{1} \operatorname{sen} \theta \\
& y_{2}=r \operatorname{sen}(\beta+\theta)=r(\cos \theta \operatorname{sen} \beta+\cos \beta \operatorname{sen} \theta)=r \cos \theta \operatorname{sen} \beta+r \cos \beta \operatorname{sen} \theta \\
& y_{2}=x_{1} \operatorname{sen} \theta+y_{1} \cos \theta
\end{aligned}
$$

Logo, de forma matricial temos:

$$
\left[\begin{array}{l}
x_{2} \\
y_{2}
\end{array}\right]=\left[\begin{array}{cc}
\cos \theta & -\operatorname{sen} \theta \\
\operatorname{sen} \theta & \cos \theta
\end{array}\right]\left[\begin{array}{l}
x_{1} \\
y_{1}
\end{array}\right]=\left[\begin{array}{c}
\cos \theta x_{1}-\operatorname{sen} \theta y_{1} \\
\operatorname{sen} \theta x_{1}+\cos \theta y_{1}
\end{array}\right]
$$

Então

$$
R_{\theta}=\left[\begin{array}{cc}
\cos \theta & -\operatorname{sen} \theta \\
\operatorname{sen} \theta & \cos \theta
\end{array}\right]
$$

Onde para $\theta=0^{\circ}$ ou $360^{\circ}$ teremos a matriz identidade que é o elemento neutro da operação multiplicação de matrizes, pois $R_{\theta} \cdot I=R_{\theta}$. Esta matriz é representada por:

$$
I=\left[\begin{array}{cc}
\cos 0 & -\operatorname{sen} 0 \\
\operatorname{sen} 0 & \cos 0
\end{array}\right]=\left[\begin{array}{ll}
1 & 0 \\
0 & 1
\end{array}\right]
$$

E se considerarmos a rotação que leva $P_{1}\left(x_{1}, y_{1}\right)$ ao ponto $P_{3}\left(x_{3}, y_{3}\right)$ no sentido horário teremos de forma análoga

$$
x_{3}=r \cos (\beta+(-\theta))=x_{1} \cos (-\theta)-y_{1} \operatorname{sen}(-\theta)=x_{1} \cos \theta+y_{1} \operatorname{sen} \theta
$$


$y_{3}=r \operatorname{sen}(\beta+(-\theta))=x_{1} \operatorname{sen}(-\theta)+y_{1} \cos (-\theta)=-x_{1} \operatorname{sen} \theta+y_{1} \cos \theta$

Logo $R_{\theta}^{-1}$ é a operação inversa de $R_{\theta}$ pois $R_{\theta} \cdot R_{\theta}^{-1}=I$ e esta é representada por:

$$
R_{\theta}^{-1}=\left[\begin{array}{cc}
\cos \theta & \operatorname{sen} \theta \\
-\operatorname{sen} \theta & \cos \theta
\end{array}\right]=R_{-\theta}
$$

\section{Verificação da representação matricial da rotação}

Dados os pontos $A=\left(x_{1}, y_{1}\right), B=\left(x_{2}, y_{2}\right), C=\left(x_{3}, y_{3}\right), D=\left(x_{4}, y_{4}\right)$, $E=\left(x_{5}, y_{5}\right), F=\left(x_{6}, y_{6}\right)$ tais que formem um hexágono regular de centro na origem do plano cartesiano, como na figura abaixo:

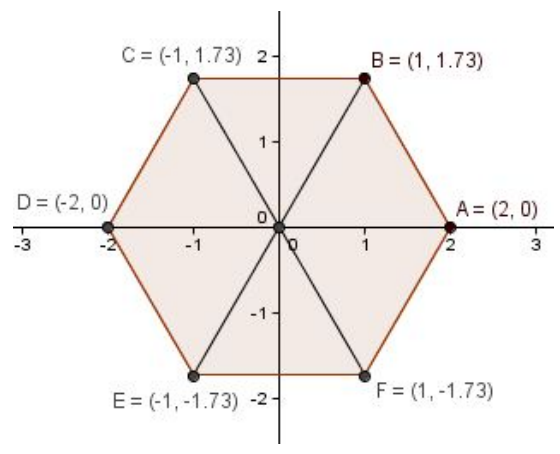

Figura 8:

Tomando a representação matricial descrita acima, $\theta=60^{\circ}$ e $A=(2,0)$ teremos

$$
\left[\begin{array}{l}
x_{2} \\
y_{2}
\end{array}\right]=\left[\begin{array}{cc}
\cos 60^{\circ} & -\operatorname{sen} 60^{\circ} \\
\operatorname{sen} 60^{\circ} & \cos 60^{\circ}
\end{array}\right]\left[\begin{array}{l}
2 \\
0
\end{array}\right]
$$

Logo $x_{2}=1$ e $y_{2}=\sqrt{3} \cong 1,73$ que são as coordenadas do ponto $\mathrm{B}$, ou seja, através da aplicação rotação de $60^{\circ}$, em sentido anti-horário, sobre o ponto A obtêm-se o ponto B e assim analogamente para os outros pontos e outros ângulos (no caso $0^{\circ}$ ou $360^{\circ}, 120^{\circ}, 180^{\circ}$ e $270^{\circ}$ ).

\section{Rotações em $\mathbb{R}^{3}$}

Seja $F\left(x_{1}, y_{1}, z_{1}\right)$ um ponto que é levado por uma rotação em torno do eixo $z$ de um ângulo $\beta$, no sentido anti-horário, no ponto $E\left(x_{2}, y_{2}, z_{2}\right)$. 


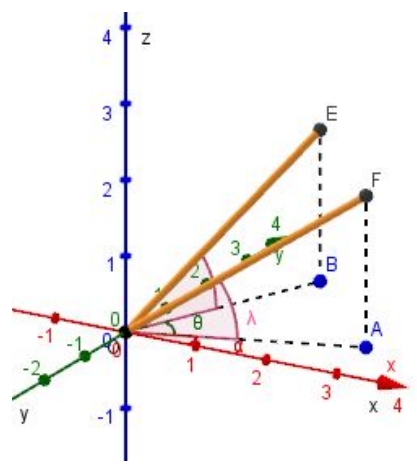

Figura 9:

Como a rotação é uma isometria então $d(O, F)=d(O, E)$ que consideremos igual a $r$ e $d(O, A)=d(O, B)=\sqrt{r^{2}-z_{1}^{2}}=s$, assim teremos;

$$
\begin{aligned}
& x_{1}=s \cdot \cos \alpha \\
& y_{1}=\operatorname{sen} \alpha \\
& z_{1}=r \cdot \operatorname{sen} \lambda \\
& \mathrm{e} \\
& x_{2}=s \cdot \cos (\alpha+\theta)=s(\cos \alpha \cdot \cos \theta-\operatorname{sen} \alpha \cdot \operatorname{sen} \theta)=s \cdot \cos \alpha \cdot \cos \theta-s \cdot \operatorname{sen} \alpha \cdot \operatorname{sen} \theta \\
& x_{2}=x_{1} \cos \theta-y_{1} \operatorname{sen} \theta \\
& y_{2}=s \cdot \operatorname{sen}(\alpha+\theta)=s(\cos \theta \operatorname{sen} \alpha+\cos \alpha \operatorname{sen} \theta)=s \cdot \cos \theta \operatorname{sen} \alpha+\operatorname{sos} \alpha \operatorname{sen} \theta \\
& y_{2}=x_{1} \operatorname{sen} \theta+y_{1} \cos \theta \\
& z_{2}=r \cdot \operatorname{sen} \lambda=z_{1}
\end{aligned}
$$

Logo, de forma matricial temos:

$$
\left[\begin{array}{l}
x_{2} \\
y_{2} \\
z_{2}
\end{array}\right]=\left[\begin{array}{ccc}
\cos \theta & -\operatorname{sen} \theta & 0 \\
\operatorname{sen} \theta & \cos \theta & 0 \\
0 & 0 & 1
\end{array}\right]\left[\begin{array}{l}
x_{1} \\
y_{1} \\
z_{1}
\end{array}\right]
$$

Então

$$
R_{z, \theta}=\left[\begin{array}{ccc}
\cos \theta & -\operatorname{sen} \theta & 0 \\
\operatorname{sen} \theta & \cos \theta & 0 \\
0 & 0 & 1
\end{array}\right]
$$

Analogamente as rotações em torno do eixo $x$ e do eixo $y$ são representadas por:

$$
R_{x, \theta}=\left[\begin{array}{ccc}
1 & 0 & 0 \\
0 & \cos \theta & -\operatorname{sen} \theta \\
0 & \operatorname{sen} \theta & \cos \theta
\end{array}\right], R_{y, \theta}=\left[\begin{array}{ccc}
\cos \theta & 0 & -\operatorname{sen} \theta \\
0 & 1 & 0 \\
\operatorname{sen} \theta & 0 & \cos \theta
\end{array}\right]
$$

E a rotação de $0^{\circ}$ ou $360^{\circ}$, é a Identidade que quando identificadas como matrizes é o elemento neutro da multiplicação de matrizes, que é representada 
por:

$$
I=\left[\begin{array}{lll}
1 & 0 & 0 \\
0 & 1 & 0 \\
0 & 0 & 1
\end{array}\right]
$$

É fácil concluir que toda rotação possui inverso, pois basta rotacionar no sentido horário e teremos:

$$
\begin{gathered}
R_{x, \theta}^{-1}=\left[\begin{array}{ccc}
1 & 0 & 0 \\
0 & \cos \theta & \operatorname{sen} \theta \\
0 & -\operatorname{sen} \theta & \cos \theta
\end{array}\right]=R_{x,-\theta}, R_{y, \theta}^{-1}=\left[\begin{array}{ccc}
\cos \theta & 0 & \operatorname{sen} \theta \\
0 & 1 & 0 \\
-\operatorname{sen} \theta & 0 & \cos \theta
\end{array}\right]=R_{y,-\theta} \\
R_{z, \theta}^{-1}=\left[\begin{array}{ccc}
\cos \theta & \operatorname{sen} \theta & 0 \\
-\operatorname{sen} \theta & \cos \theta & 0 \\
0 & 0 & 1
\end{array}\right]=R_{z,-\theta}
\end{gathered}
$$

\section{Verificação da representação matricial da rotação}

Dados os pontos $I=\left(x_{1}, y_{1}, z_{1}\right), J=\left(x_{2}, y_{2}, z_{2}\right), K=\left(x_{3}, y_{3}, z_{3}\right), L=$ $\left(x_{4}, y_{4}, z_{4}\right), M=\left(x_{5}, y_{5}, z_{5}\right), O=\left(x_{6}, y_{6}, z_{6}\right), P=\left(x_{7}, y_{7}, z_{7}\right), Q=\left(x_{8}, y_{8}, z_{8}\right)$, $R=\left(x_{9}, y_{9}, z_{9}\right), S=\left(x_{10}, y_{10}, z_{10}\right), T=\left(x_{11}, y_{11}, z_{11}\right), U=\left(x_{12}, y_{12}, z_{12}\right)$ tais que formem um prisma hexagonal regular de centro na origem do sistema cartesiano, conforme a figura.

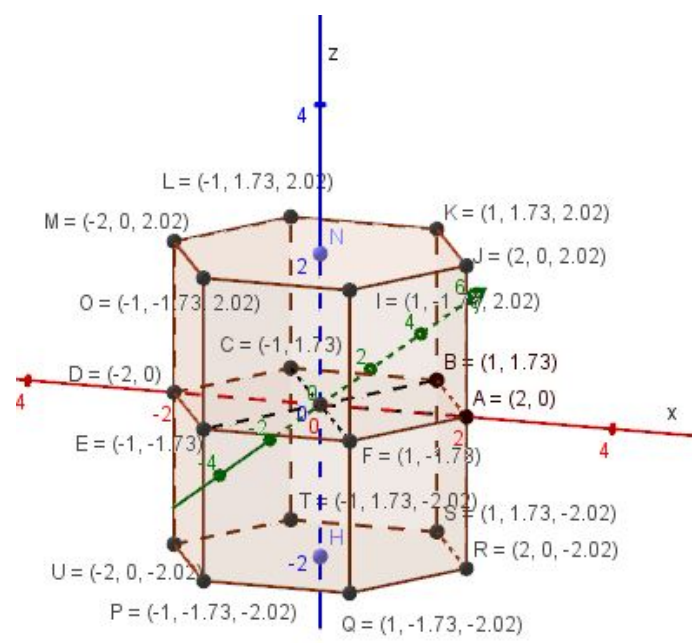

Figura 10:

Tomando a representação matricial descrita acima, $\theta=180^{\circ}$ em torno do eixo $x$ e $\mathrm{J}=(2,0,2.02)$ teremos 


$$
\left[\begin{array}{l}
x_{9} \\
y_{9} \\
z_{9}
\end{array}\right]=\left[\begin{array}{ccc}
1 & 0 & 0 \\
0 & \cos 180^{\circ} & -\operatorname{sen} 180^{\circ} \\
0 & \operatorname{sen} 180^{\circ} & \cos 180^{\circ}
\end{array}\right]\left[\begin{array}{c}
2 \\
0 \\
2.02
\end{array}\right]
$$

Logo $x_{9}=2, y_{9}=0$ e $z_{9}=-2.02$ que são as coordenadas do ponto $R$, ou seja, através da aplicação da rotação de $180^{\circ}$ em torno do eixo $x$, em sentido anti-horário, sobre o ponto $J$ obtêm-se o ponto $R$ e assim analogamente para os outros pontos, outros ângulos e outros eixos.

\subsubsection{Reflexões}

\section{Reflexões em $\mathbb{R}$}

Na reta $\mathbb{R}$ uma reflexão $r: \mathbb{R} \rightarrow \mathbb{R}$ é definida por um de seus pontos, exatamente o que é fixado pela reflexão, isto é, $r(P)=P$ e para $X \neq P$ tal que $r(X)=X^{\prime} \neq X$ e $d(P, X)=d\left(P, X^{\prime}\right)$.

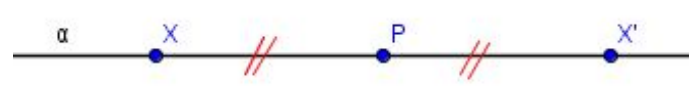

Figura 11:

\section{Reflexões em $\mathbb{R}^{2}$}

No plano $\mathbb{R}^{2}$ uma reflexão $r: \mathbb{R}^{2} \rightarrow \mathbb{R}^{2}$ é definida por uma de suas retas, exatamente a reta $m$ onde a reflexão é fixa, isto é, se $P \in m$ então $r(P)=P$. Se $P_{2} \notin m$ então $r\left(P_{2}\right)=P_{1}$ onde $d\left(P_{2}, m\right)=d\left(P_{1}, m\right)$ e além disso $P_{2}$ e $P_{1}$ pertencem a mesma reta $\alpha$ (perpendicular a $m$ ).

Seja $P_{1}\left(x_{1}, y_{1}\right)$ um ponto que é levado por uma reflexão em torno da reta que passa pela origem $y=m x$ (onde $m=\operatorname{tg} \beta$ ) no ponto $P_{2}\left(x_{2}, y_{2}\right)$. 


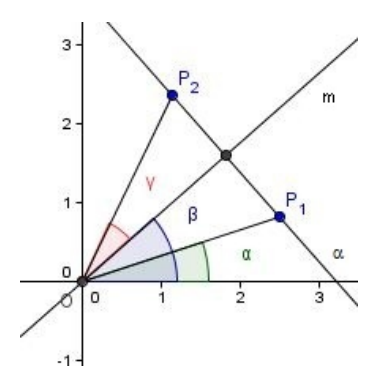

Figura 12:

Considerando $d\left(O, P_{1}\right)=d\left(O, P_{2}\right)=r$, temos:

$x_{1}=r \cos \alpha$

$y_{1}=r \operatorname{sen} \alpha$

e como $\gamma=\beta-\alpha$

$x_{2}=r \cos (2 \beta-\alpha)=r(\cos 2 \beta \cos \alpha+\operatorname{sen} 2 \beta \operatorname{sen} \alpha)=x_{1} \cos 2 \beta+y_{1} \operatorname{sen} 2 \beta$

$y_{2}=r \operatorname{sen}(2 \beta-\alpha)=r(\cos \alpha \operatorname{sen} 2 \beta-\cos 2 \beta \operatorname{sen} \alpha)=x_{1} \operatorname{sen} 2 \beta-y_{1} \cos 2 \beta$

De forma matricial temos:

$$
\left[\begin{array}{l}
x_{2} \\
y_{2}
\end{array}\right]=\left[\begin{array}{cc}
\cos 2 \beta & \operatorname{sen} 2 \beta \\
\operatorname{sen} 2 \beta & -\cos 2 \beta
\end{array}\right]\left[\begin{array}{l}
x_{1} \\
y_{1}
\end{array}\right]
$$

onde

$$
r=\left[\begin{array}{cc}
\cos 2 \beta & \operatorname{sen} 2 \beta \\
\operatorname{sen} 2 \beta & -\cos 2 \beta
\end{array}\right]
$$

Usando os conceitos e as propriedades de matrizes e transformações poderemos obter a matriz reflexão em relação a uma reta $\mathrm{r}$ (eixo de simetria).

Primeiramente, dado um ponto a reflexão em torno do eixo $x$ leva o ponto ao seu simétrico, ou seja, $r: \mathbb{R}^{2} \rightarrow \mathbb{R}^{2}, r(x, y)=(x,-y)$, e analogamente em torno do eixo y, $r(x, y)=(-x, y)$.

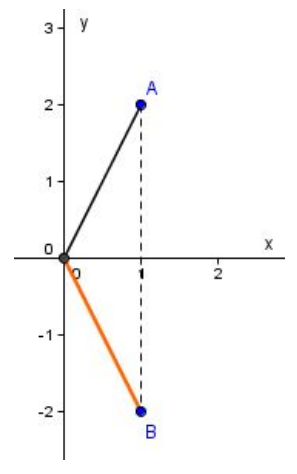

Figura 13:

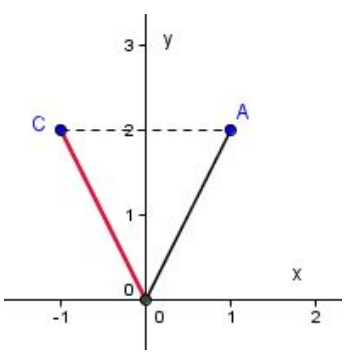

Figura 14: 
De forma matricial teremos:

$$
r_{x}\left(\left[\begin{array}{l}
x \\
y
\end{array}\right]\right)=\left[\begin{array}{cc}
1 & 0 \\
0 & -1
\end{array}\right]\left[\begin{array}{l}
x \\
y
\end{array}\right] \text { e } r_{y}\left(\left[\begin{array}{l}
x \\
y
\end{array}\right]\right)=\left[\begin{array}{cc}
-1 & 0 \\
0 & 1
\end{array}\right]\left[\begin{array}{l}
x \\
y
\end{array}\right]
$$

Logo, as representações matriciais da reflexão em torno do eixo $x, r_{x}$ e em torno do eixo $y, r_{y}$ são dadas por:

$$
r_{x}=\left[\begin{array}{cc}
1 & 0 \\
0 & -1
\end{array}\right] \text { e } r_{y}=\left[\begin{array}{cc}
-1 & 0 \\
0 & 1
\end{array}\right]
$$

Agora considere uma reflexão de um ponto $A$ em torno de uma reta $r$ que passa pela origem e que não seja nenhum dos eixos $(x, y$ ou $z)$. Aparentemente essa reflexão não é tão simples mas se rotacionarmos $r$ até o eixo $x$ (ou eixo $y$ ) e o ponto no mesmo ângulo e sentido, em seguida aplicarmos a reflexão do ponto em torno do eixo e rotacionarmos a reta e o ponto refletido novamente ao sistema inicial teremos a reflexão pretendida, assim:

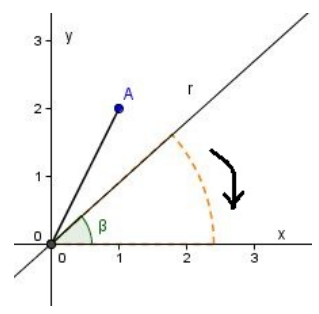

Figura 15:

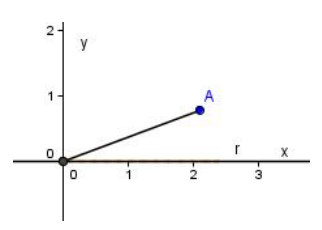

Figura 16:

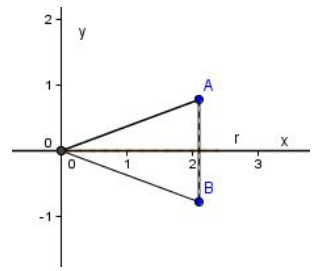

Figura 17:

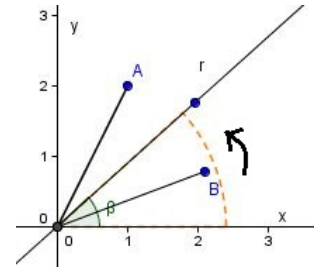

Figura 18:

Matricialmente temos:

$$
\begin{gathered}
R_{\beta} r_{x} R_{\beta}^{-1}=\left[\begin{array}{cc}
\cos \beta & -\operatorname{sen} \beta \\
\operatorname{sen} \beta & \cos \beta
\end{array}\right]\left[\begin{array}{cc}
1 & 0 \\
0 & -1
\end{array}\right]\left[\begin{array}{cc}
\cos \beta & \operatorname{sen} \beta \\
-\operatorname{sen} \beta & \cos \beta
\end{array}\right]= \\
{\left[\begin{array}{cc}
\cos 2 \beta & \operatorname{sen} 2 \beta \\
\operatorname{sen} 2 \beta & -\cos 2 \beta
\end{array}\right]}
\end{gathered}
$$

De forma análoga teremos a reflexão do ponto através da rotação da reta $r$ até o eixo $y$.

$$
\begin{gathered}
R_{\beta}^{-1} r_{y} R_{\beta}=\left[\begin{array}{cc}
\cos \beta & \operatorname{sen} \beta \\
-\operatorname{sen} \beta & \cos \beta
\end{array}\right]\left[\begin{array}{cc}
-1 & 0 \\
0 & 1
\end{array}\right]\left[\begin{array}{cc}
\cos \beta & -\operatorname{sen} \beta \\
\operatorname{sen} \beta & \cos \beta
\end{array}\right]= \\
{\left[\begin{array}{cc}
-\cos 2 \beta & \operatorname{sen} 2 \beta \\
\operatorname{sen} 2 \beta & \cos 2 \beta
\end{array}\right]}
\end{gathered}
$$

onde $\beta$ é o ângulo formado entre a reta $r$ e o eixo referido ( $x$ ou $y$ ). 


\section{Verificação da representação matricial da reflexão}

Dados os pontos $A=\left(x_{1}, y_{1}\right), B=\left(x_{2}, y_{2}\right), C=\left(x_{3}, y_{3}\right), D=\left(x_{4}, y_{4}\right)$, $E=\left(x_{5}, y_{5}\right), F=\left(x_{6}, y_{6}\right)$ tais que formem um hexágono regular de centro na origem do plano cartesiano, como no exemplo.

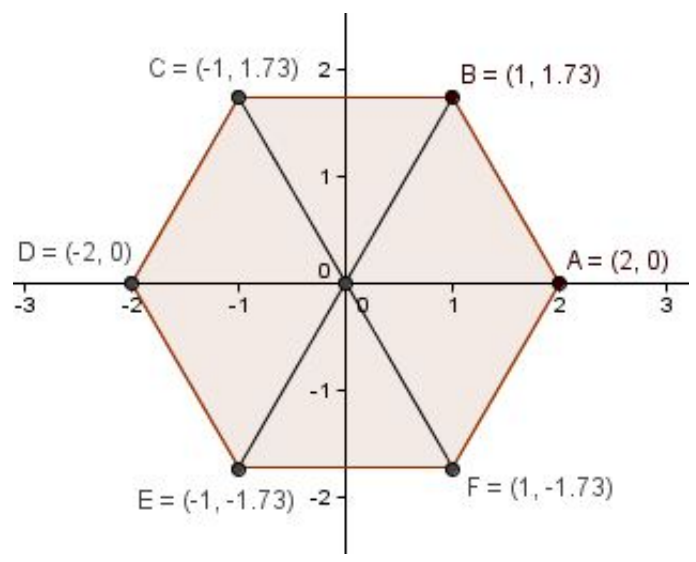

Figura 19:

Tomando a representação matricial descrita acima, $\beta=120^{\circ}$ e $A=(2,0)$ teremos

$$
\left[\begin{array}{l}
x_{5} \\
y_{5}
\end{array}\right]=\left[\begin{array}{cc}
\cos 240^{\circ} & \operatorname{sen} 240^{\circ} \\
\operatorname{sen} 240^{\circ} & -\cos 240^{\circ}
\end{array}\right]\left[\begin{array}{l}
2 \\
0
\end{array}\right]
$$

Logo $x_{5}=-1$ e $y_{5}=-\sqrt{3} \cong-1,73$ que são as coordenadas do ponto $E$, ou seja, através da aplicação de reflexão do ponto $A$ sobre a reta $C F$ (eixo de simetria) com inclinação de $120^{\circ}$ em relação ao eixo $x$, obtêm-se o ponto $E$ e assim analogamente para os outros pontos e outros eixos de simetria.

\section{Reflexões em $\mathbb{R}^{3}$}

Dado um ponto em $\mathbb{R}^{3}$, a reflexão em torno do plano $x O y$ leva o ponto ao seu simétrico, ou seja, $r: \mathbb{R}^{3} \rightarrow \mathbb{R}^{3}, r_{x y}(x, y, z)=(x, y,-z)$, analogamente em torno do plano $y O z, r_{y z}(x, y, z)=(-x, y, z)$ e em torno do plano $x O z$, $r_{x z}(x, y, z)=(x,-y, z)$.

Matricialmente:

$$
r_{x y}=\left[\begin{array}{ccc}
1 & 0 & 0 \\
0 & 1 & 0 \\
0 & 0 & -1
\end{array}\right] r_{y z}=\left[\begin{array}{ccc}
-1 & 0 & 0 \\
0 & 1 & 0 \\
0 & 0 & 1
\end{array}\right] r_{x z}=\left[\begin{array}{ccc}
1 & 0 & 0 \\
0 & -1 & 0 \\
0 & 0 & 1
\end{array}\right]
$$


Seja $F\left(x_{1}, y_{1}, z_{1}\right)$ um ponto que é levado por uma reflexão em relação ao plano $z m$, passando pela origem do sistema cartesiano e formando com o plano $x z$ um ângulo $\beta$, no ponto $K\left(x_{2}, y_{2}, z_{2}\right)$. Ao rotacionarmos o plano $z m$ até o plano $x z$ e o ponto no mesmo ângulo e sentido, aplicarmos a reflexão do ponto em relação ao plano $x z$ e em seguida rotacionarmos o plano $z m$ e o ponto a posição inicial, obteremos a reflexão pretendida.

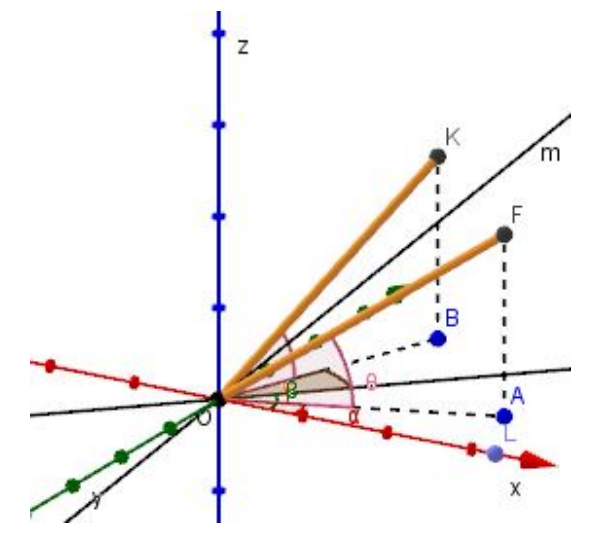

Figura 20:

Matricialmente

$$
\left[\begin{array}{l}
x_{2} \\
y_{2} \\
z_{2}
\end{array}\right]=\left[\begin{array}{ccc}
\cos 2 \beta & \operatorname{sen} 2 \beta & 0 \\
\operatorname{sen} 2 \beta & -\cos 2 \beta & 0 \\
0 & 0 & 1
\end{array}\right]\left[\begin{array}{l}
x_{1} \\
y_{1} \\
z_{1}
\end{array}\right]
$$

$\log O$

$$
r_{z m}=\left[\begin{array}{ccc}
\cos 2 \beta & \operatorname{sen} 2 \beta & 0 \\
\operatorname{sen} 2 \beta & -\cos 2 \beta & 0 \\
0 & 0 & 1
\end{array}\right]
$$

Analogamnente as reflexões em torno dos planos $x m$ e ym são representadas por:

$$
r_{x m}=\left[\begin{array}{ccc}
1 & 0 & 0 \\
0 & \cos 2 \beta & \operatorname{sen} 2 \beta \\
0 & \operatorname{sen} 2 \beta & -\cos 2 \beta
\end{array}\right], r_{y m}=\left[\begin{array}{ccc}
\cos 2 \beta & 0 & \operatorname{sen} 2 \beta \\
0 & 1 & 0 \\
\operatorname{sen} 2 \beta & 0 & -\cos 2 \beta
\end{array}\right]
$$




\section{Conceitos Algébricos Fundamentais}

\subsection{Grupos}

Dizemos que * é uma operação binária definida num conjunto $\mathbb{G}$ não vazio, ou é uma lei de composição em $\mathbb{G}$, se para quaisquer $x, y \in \mathbb{G}$, isto é, para qualquer par ordenado $(x, y)$ de $\mathbb{G} X \mathbb{G}$, corresponde um único elemento de $\mathbb{G}$, denotado por $z=x * y$ :

$$
\text { *: } \begin{aligned}
\mathbb{G} X \mathbb{G} & \rightarrow \mathbb{G} \\
(x, y) & \mapsto z
\end{aligned}
$$

Um grupo $\mathbb{G}$ é um conjunto não vazio munido de uma operação binária $*$, denotado $(\mathbb{G}, *)$, que satisfaz

i. $a *(b * c)=(a * b) * c$, para todo a, b e c $\in \mathbb{G}$ (associativa).

ii. existe um elemento $e \in \mathbb{G}$ tal que $a * e=a$ e $e * a=a$, para todo $a \in \mathbb{G}$ (elemento neutro)

iii. para cada $a \in \mathbb{G}$ existe em elemento $d \in \mathbb{G}$ tal que $a * d=e$ e $d * a=e$ (elemento inverso).

O grupo é chamado abeliano se para todo $a, b \in \mathbb{G}, a * b=b * a$ (comutativa).

\section{Exemplos de Grupos}

Considere o conjunto numérico $\mathbb{Z}$ e as classes módulo $7(\overline{0}, \overline{1}, \overline{2}, \overline{3}, \overline{4}$, $\overline{5}, \overline{6})$ que por facilidade denotaremos nas tabelas abaixo sem o traço e observe as tabelas obtidas da operações adição e multiplicação módulo 7.

\begin{tabular}{c|ccccccc}
+ & 0 & 1 & 2 & 3 & 4 & 5 & 6 \\
\hline 0 & 0 & 1 & 2 & 3 & 4 & 5 & 6 \\
1 & 1 & 2 & 3 & 4 & 5 & 6 & 0 \\
2 & 2 & 3 & 4 & 5 & 6 & 0 & 1 \\
3 & 3 & 4 & 5 & 6 & 0 & 1 & 2 \\
4 & 4 & 5 & 6 & 0 & 1 & 2 & 3 \\
5 & 5 & 6 & 0 & 1 & 2 & 3 & 4 \\
6 & 6 & 0 & 1 & 2 & 3 & 4 & 5
\end{tabular}




\begin{tabular}{c|ccccccc}
$\mathrm{x}$ & 0 & 1 & 2 & 3 & 4 & 5 & 6 \\
\hline 0 & 0 & 0 & 0 & 0 & 0 & 0 & 0 \\
1 & 0 & 1 & 2 & 3 & 4 & 5 & 6 \\
2 & 0 & 2 & 4 & 6 & 1 & 3 & 5 \\
3 & 0 & 3 & 6 & 2 & 5 & 1 & 4 \\
4 & 0 & 4 & 1 & 5 & 2 & 6 & 3 \\
5 & 0 & 5 & 3 & 1 & 6 & 4 & 2 \\
6 & 0 & 6 & 5 & 4 & 3 & 2 & 1
\end{tabular}

A partir das observações da tabela é fácil verificar que as propriedades de grupo são satisfeitas em $\left(\mathbb{Z}_{7},+\right)$, mas nem todas são satisfeitas em $\left(\mathbb{Z}_{7},.\right)$, pois o 0 (zero) não possui elemento inverso uma vez que, para esta operação, 1 é o elemento neutro. Assim podemos afirmar que o conjunto $\mathbb{Z}_{7}$ definida na operação adição é um grupo mas o mesmo conjunto definida na operação multiplicação não é.

\subsubsection{Grupo de Matrizes (com a operação multiplicação)}

O conjunto das matrizes $n \times n$ inversíveis, ou seja, com determinante não nulo, munido da operação de multiplicação de matrizes é um grupo. Denominaremos este grupo de matrizes por $G L(n, \mathbb{R})$.

\section{Exemplos de Grupo de Matrizes}

Dadas as matrizes

$$
\left[\begin{array}{ll}
a_{11} & a_{12} \\
a_{21} & a_{22}
\end{array}\right]
$$

que pertencem ao conjunto das matrizes $2 \times 2$ com determinante diferente de zero, fica fácil verificar que as propriedades de grupo são satisfeitas pois também são propriedades de produto de matrizes, como descrito abaixo:

$$
\begin{gathered}
{\left[\begin{array}{ll}
a_{11} & a_{12} \\
a_{21} & a_{22}
\end{array}\right] \cdot\left[\begin{array}{ll}
1 & 0 \\
0 & 1
\end{array}\right]=\left[\begin{array}{ll}
a_{11} & a_{12} \\
a_{21} & a_{22}
\end{array}\right]} \\
\left(\left[\begin{array}{ll}
a_{11} & a_{12} \\
a_{21} & a_{22}
\end{array}\right] \cdot\left[\begin{array}{ll}
b_{11} & b_{12} \\
b_{21} & b_{22}
\end{array}\right]\right) \cdot\left[\begin{array}{ll}
c_{11} & c_{12} \\
c_{21} & c_{22}
\end{array}\right]= \\
{\left[\begin{array}{ll}
a_{11} & a_{12} \\
a_{21} & a_{22}
\end{array}\right] \cdot\left(\left[\begin{array}{ll}
b_{11} & b_{12} \\
b_{21} & b_{22}
\end{array}\right] \cdot\left[\begin{array}{ll}
c_{11} & c_{12} \\
c_{21} & c_{22}
\end{array}\right]\right)}
\end{gathered}
$$


Como por definição o determinante é diferente de zero então existe uma matriz tal que:

$$
\left[\begin{array}{ll}
a_{11} & a_{12} \\
a_{21} & a_{22}
\end{array}\right] \cdot\left[\begin{array}{ll}
d_{11} & d_{12} \\
d_{21} & d_{22}
\end{array}\right]=\left[\begin{array}{ll}
1 & 0 \\
0 & 1
\end{array}\right]
$$

Assim, dizemos que o conjunto $G L(2)$ munido da operação multiplicação é um grupo.

\subsubsection{Grupo de Permutações}

Dado um conjunto $A$ de $n$ elementos representado por $A=\{1, \ldots, n\}$, então toda bijeção $\sigma: A \rightarrow A$ é chamada uma permutação de $A$, que podemos escrever na forma matricial como a que se segue:

$$
\sigma=\left[\begin{array}{cccc}
1 & 2 & \cdots & n \\
\sigma(1) & \sigma(2) & \cdots & \sigma(n)
\end{array}\right]
$$

onde $\sigma(i)(\mathrm{i}=1, \ldots, \mathrm{n})$ é a imagem de cada $i$ pela aplicação $\sigma$ e a ordem deste conjunto de bijeções, denotado $\operatorname{Bij}(A)$ é $n$ !.

\section{Exemplo de Grupo de Permutações}

Dado um conjunto de 3 elementos $B=\{1,2,3\}$, a aplicação $\sigma: B \rightarrow B$ tal que $\sigma(1)=3, \sigma(2)=1, \sigma(3)=2$ é uma bijeção. E todas as possíveis bijeções $\sigma: B \rightarrow B$ são:

$$
\begin{aligned}
& \sigma_{1}=\left[\begin{array}{lll}
1 & 2 & 3 \\
1 & 2 & 3
\end{array}\right] \sigma_{2}=\left[\begin{array}{lll}
1 & 2 & 3 \\
1 & 3 & 2
\end{array}\right] \sigma_{3}=\left[\begin{array}{lll}
1 & 2 & 3 \\
2 & 1 & 3
\end{array}\right] \\
& \sigma_{4}=\left[\begin{array}{lll}
1 & 2 & 3 \\
3 & 2 & 1
\end{array}\right] \sigma_{5}=\left[\begin{array}{lll}
1 & 2 & 3 \\
3 & 1 & 2
\end{array}\right] \quad \sigma_{6}=\left[\begin{array}{lll}
1 & 2 & 3 \\
2 & 3 & 1
\end{array}\right]
\end{aligned}
$$

Logo $\operatorname{Bij}(B)$, usualmente chamado $P_{3}=\left\{\sigma_{1}, \sigma_{2}, \sigma_{3}, \sigma_{4}, \sigma_{5}, \sigma_{6}\right\}$.

O conjunto de todas estas permutações munido da operação composição de funções é um grupo.

\subsubsection{Homomorfismo de Grupos}

Dados dois grupos $(\mathbb{S},$.$) e (\mathbb{G}, *)$. Uma aplicação $\sigma: \mathbb{S} \rightarrow \mathbb{G}$ é chamada de homomorfismo de grupos, se e somente se, $\sigma(a . b)=\sigma(a) * \sigma(b)$, para quaisquer $a, b \in \mathbb{S}$.

Um homomorfismo é sobrejetivo se $\sigma(\mathbb{S})=\mathbb{G}$ e é injetivo se para quaisquer $a$ e $b \in \mathbb{S}, \sigma(a)=\sigma(b)$ então $a=b$. Quando um homorfismo é sobrejetivo e injetivo dizemos que o homomorfismo é bijetor.

Obs: $\sigma$ é injetivo se, e somente se, $\sigma^{-1}\left\{e^{\prime}\right\}=\{e\}$, onde $e^{\prime}$ e $e$ são elementos neutro. 


\subsubsection{Isomorfismo de Grupos}

O homorfismo é chamado isomorfismo se for bijetor.

\subsubsection{Grupos Isomorfos}

Se a aplicação $\sigma: \mathbb{S} \rightarrow \mathbb{G}$ é um isomor fismo de grupos então $S$ e $G$ são chamados isomorfos.

\section{Exemplos de Grupos Isomorfos}

Dados os grupos $\left(\mathbb{R}_{+}\right.$, . ) e $(\mathbb{R},+)$, então a aplicação

$$
\begin{aligned}
\log : \mathbb{R}_{+} & \rightarrow \mathbb{R} \\
x & \mapsto \log (x)
\end{aligned}
$$

é um homorfismo de grupos pois $\log (x . y)=\log x+\log y$, para quaisquer $x, y \in \mathbb{R}$

Note que a aplicação log leva elemento neutro de $\left(\mathbb{R}_{+}\right.$, . $)$no elemento neutro de $(\mathbb{R},+)(\log (1)=0)$ e como a aplicação inverva de $\log$ :

$$
\begin{aligned}
\exp : \mathbb{R} & \rightarrow \mathbb{R}_{+} \\
x & \mapsto \exp (x)
\end{aligned}
$$

também é um homorfismo, já que $\exp (x . y)=\exp (x)+\exp (y)$, para quaisquer $x, y \in \mathbb{R}$

Podemos concluir que a aplicação log é um isomorfismo de grupos e assim $\left(\mathbb{R}_{+},.\right)$e $(\mathbb{R},+)$ são isomorfos. 


\section{Grupos de Simetrias de Figuras Planas}

Dada uma figura geométrica $F$, uma transformação bijetora $T: F \rightarrow F$ que preserva distâncias entre os pontos de $F$ é uma simetria de $F$.

Observe que dada duas simetrias $T_{1}: F \rightarrow F$ e $T_{2}: F \rightarrow F$ a composição $T_{2} \circ T_{1}: F \rightarrow F$ é bijetora e preserva distâncias, logo ainda é uma simetria.

É claro que, $I: F \rightarrow F$, a aplicação identidade é uma simetria. E se $T$ é uma simetria, então existe a aplicação $T^{-1}$ inversa de $T$ tal que $d\left(T^{-1}(a), T^{-1}(b)\right)=d(a, b)$ satisfazendo assim as propriedades de grupo.

Logo, o conjunto de todas as transformações bijetoras de $F$ em $F$, que preserva a distância entre seus pontos, munido da operação composição de funções forma o grupo de simetria da figura.

Para ter uma ideia geométrica estudaremos o grupo de simetria de algumas figuras planas.

\subsection{Grupo de Simetrias de um Triângulo Isósceles}

Tomemos o triângulo isósceles de vértices $A, B$ e $C$ conforme o desenho, onde $y$ é mediatriz de $B C$.

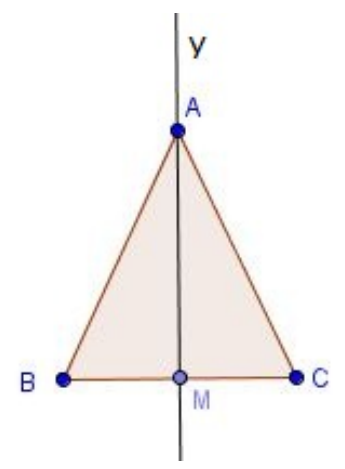

Figura 21:

A rotação de $0^{\circ}$ em torno do centro do triângulo é determinada pela bijeção dos vértices do triângulo.

$$
I=\left[\begin{array}{lll}
A & B & C \\
A & B & C
\end{array}\right]
$$

A reflexão $r: \mathbb{R}^{2} \rightarrow \mathbb{R}^{2}$, fixa a reta $y$ e leva $B$ em $C$ e $C$ em $B$ de tal forma que se tenha uma isometria já que $d(B, M)=d(C, M)$. Essa reflexão é determinada por:

$$
r=\left[\begin{array}{lll}
A & B & C \\
A & C & B
\end{array}\right]
$$


Esses movimentos formam um conjunto $\{I, r\}$ que denominaremos por $\mathbb{S}_{t i}$

Acompanhando a tabela abaixo de operação composição dessas transformações no plano

\begin{tabular}{c|cc}
$\mathrm{o}$ & $\mathrm{I}$ & $r_{d}$ \\
\hline $\mathrm{I}$ & $\mathrm{I}$ & $r_{d}$ \\
$r_{d}$ & $r_{d}$ & $\mathrm{I}$
\end{tabular}

É fácil concluir que as três propriedades de grupo com a operação composição são satisfeitas e então o conjunto $\mathbb{S}_{t i}$ é denomidado grupo de simetria do triângulo isósceles.

\subsection{Grupo de Simetrias de um Retângulo}

Consideremos o retângulo (não quadrado) $A B C D$, onde $x$ e $y$ são mediatrizes dos lados $A D$ e $D C$

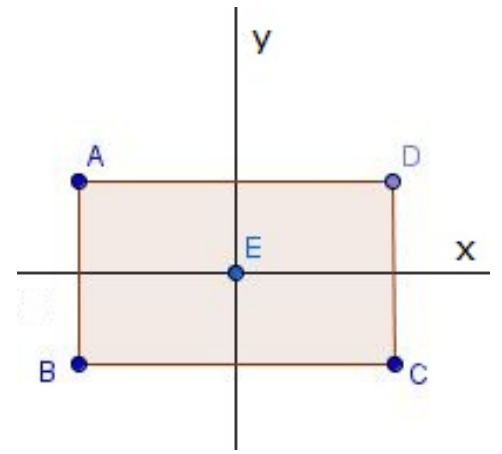

Figura 22:

Os movimentos de reflexão em torno dos eixos de coordenadas $x$ e $y$ são simetrias e são descritas pelo esquema

$$
r_{y}=\left[\begin{array}{llll}
A & B & C & D \\
D & C & B & A
\end{array}\right] r_{x}=\left[\begin{array}{llll}
A & B & C & D \\
B & A & D & C
\end{array}\right]
$$

Se rotacionarmos $0^{\circ}, 180^{\circ}$ em torno de $E$ (centro do retângulo) no sentido anti-horário, teremos

$$
I=\left[\begin{array}{llll}
A & B & C & D \\
A & B & C & D
\end{array}\right] \quad R_{180}=\left[\begin{array}{cccc}
A & B & C & D \\
C & D & A & B
\end{array}\right]
$$

Esses movimentos formam um conjunto que denominaremos 
$\mathbb{S}_{r}=\left\{I, r_{y}, r_{x}, R_{180}\right\}$.

Acompanhando a tabela abaixo de operação composição dessas transformações no plano

\begin{tabular}{c|cccc}
$\circ$ & $I$ & $r_{y}$ & $r_{x}$ & $R_{180}$ \\
\hline$I$ & $I$ & $r_{y}$ & $r_{x}$ & $R_{180}$ \\
$r_{y}$ & $r_{y}$ & $I$ & $R_{180}$ & $r_{x}$ \\
$r_{x}$ & $r_{x}$ & $R_{180}$ & $I$ & $r_{y}$ \\
$R_{180}$ & $R_{180}$ & $r_{x}$ & $r_{y}$ & $I$
\end{tabular}

observamos que:

$r_{y}{ }^{\mathrm{o}}\left(r_{x}{ }^{\mathrm{o}} R_{180}\right)=r_{y}{ }^{\mathrm{o}} r_{y}=r_{x}=R_{180}{ }^{\circ} R_{180}=\left(r_{y}{ }^{\mathrm{o}} r_{x}\right)^{\circ} R_{180}$ para quaisquer três elementos do conjunto $\mathbb{S}_{r}$.

$r_{y}{ }^{\circ} I=r_{y}, \ldots, R_{180}{ }^{\circ} I=R_{180}$, então $I$ ( elemento neutro) pertence a $\mathbb{S}_{r}$.

$r_{y}{ }^{\circ} R_{180}=I, \ldots, r_{x}{ }^{\circ} r_{x}$, assim cada elemento do conjunto $\mathbb{S}_{r}$ possui inverso.

Além disso, analisando a tabela vemos que é simétrica implicando que $r_{x}{ }^{\circ} r_{y}=r_{y}{ }^{\circ} r_{x}$, ou seja, satisfaz a propriedade comutativa.

Logo o conjunto $\mathbb{S}_{r}$ munido da operação composição é o grupo de simetrias do retângulo e esse é comutativo.

\subsection{Grupo de Simetrias de um Triângulo Equilátero}

Dado um triângulo equilátero $A B C$, temos:

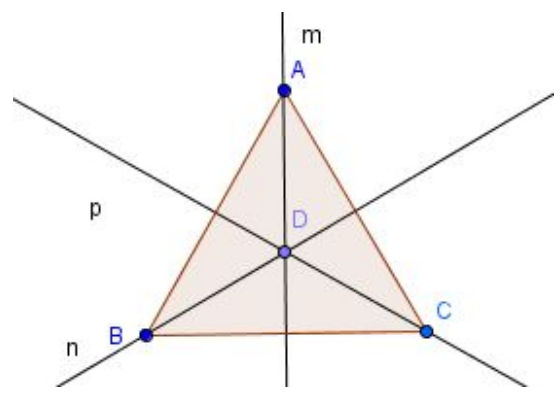

Figura 23:

$$
r_{m}=\left[\begin{array}{ccc}
A & B & C \\
A & C & B
\end{array}\right] r_{n}=\left[\begin{array}{ccc}
A & B & C \\
C & B & A
\end{array}\right] r_{p}=\left[\begin{array}{ccc}
A & B & C \\
B & A & C
\end{array}\right]
$$

onde $r_{m}, r_{n}$ e $r_{p}$ são os movimentos de reflexão em torno do eixo de simetria axial, ou seja das retas $m, n$ e $p$.

Ao rotacionarmos $0^{\circ}, 120^{\circ}$ e $240^{\circ}$ em torno de $D$ (centro do triângulo equilátero) no sentido anti-horário, teremos: 


$$
I=\left[\begin{array}{ccc}
A & B & C \\
A & B & C
\end{array}\right] R_{120^{\circ}}=\left[\begin{array}{ccc}
A & B & C \\
C & A & B
\end{array}\right] \quad R_{240^{\circ}}=\left[\begin{array}{ccc}
A & B & C \\
B & C & A
\end{array}\right]
$$

Logo o grupo de simetrias do triângulo equilátero é $\mathbb{D}_{3}=\left\{I, r_{m}, r_{n}, r_{p}, R_{1}, R_{2}\right\}$.

Para facilitar nosso entendimento para futuras análises tomaremos $a=$ $R_{120^{\circ}}$ e $b=r_{n}$ e então obteremos, pela operação composição, $a^{2}=R_{240^{\circ}}$, $a^{3}=R_{360^{\circ}}=I, a b=r_{p}, a^{2} b=r_{m}, a^{3} b=r_{n}$.

Assim fica fácil concluir que para determinarmos o grupo de simetrias de figuras geometricas planas e limitadas basta operarmos as rotações $n$ vezes, onde $n$ é o número de lados e em seguida através de uma única reflexão operada com essas rotações serão obtidas as outras reflexões.

Logo o grupo de simetria pode ser descrito como $\mathbb{D}_{3}=\left\{I, a, a^{2}, b, a b, a^{2} b\right\}$ e podemos notar que o grupo de simetrias do triângulo equilátero é isomorfo ao grupo de permutações do conjunto $\{A, B, C\}$.

\subsection{Grupo de Simetrias do Quadrado}

Considere um quadrado $A B C D$, onde $x$ e $y$ são mediatrizes dos lados $A D$ e $D C$, respectivamente, $g$ e $h$ são as diagonais do quadrado.

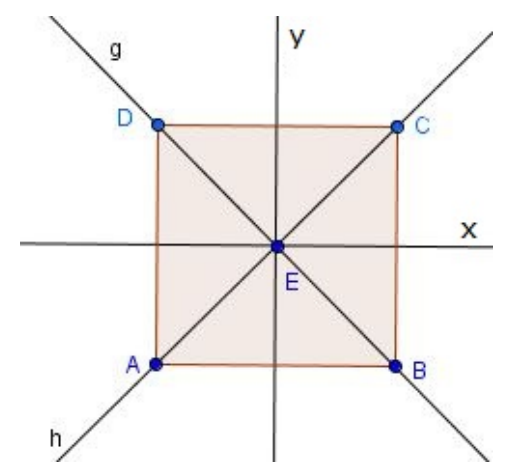

Figura 24:

Os movimentos de reflexão em torno dos eixos de simetria axial são mostrados pelo esquema

$$
\begin{aligned}
& r_{y}=\left[\begin{array}{llll}
A & B & C & D \\
B & A & D & C
\end{array}\right] r_{x}=\left[\begin{array}{llll}
A & B & C & D \\
D & C & B & A
\end{array}\right] \\
& r_{g}=\left[\begin{array}{llll}
A & B & C & D \\
C & B & A & D
\end{array}\right] r_{h}=\left[\begin{array}{llll}
A & B & C & D \\
A & D & C & B
\end{array}\right]
\end{aligned}
$$


Se rotacionarmos $0^{\circ}, 90^{\circ}, 180^{\circ}$ e $270^{\circ}$ em torno de $E$ (centro do quadrado) no sentido anti-horário, teremos

$$
\begin{gathered}
I=\left[\begin{array}{cccc}
A & B & C & D \\
A & B & C & D
\end{array}\right] R_{90^{\circ}}=\left[\begin{array}{cccc}
A & B & C & D \\
D & A & B & A
\end{array}\right] \\
R_{180^{\circ}}=\left[\begin{array}{llll}
A & B & C & D \\
C & D & A & B
\end{array}\right] R_{270^{\circ}}=\left[\begin{array}{llll}
A & B & C & D \\
B & C & D & A
\end{array}\right]
\end{gathered}
$$

Logo essas transformações formam o grupo de simetria do quadrado que denominaremos $\mathbb{D}_{4}=\left\{I, a, a^{2}, a^{3}, b, a b, a^{2} b, a^{3} b\right\}$, onde $a=R_{90^{\circ}}, a^{2}=R_{180^{\circ}}$, $a^{3}=R_{270^{\circ}}$ e $b$ é uma reflexão do quadrado. $\mathbb{D}_{4}$ é um subgrupo do grupo de permutações do conjunto $\{A, B, C, D\}$, e é fácil observar que, por exemplo, a bijeção a seguir não preserva a figura invariante no plano, ou seja, não é uma isometria.

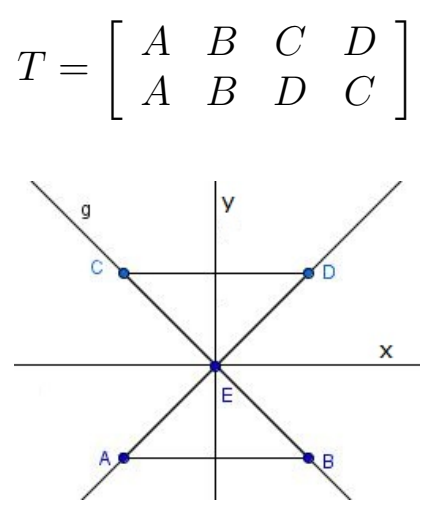

Figura 25:

\subsection{Grupo de Simetrias do Pentágono Regular}

Seja o pentágono $A B C D E$, onde $g, j, f, i$ e $h$ são mediatrizes dos lados $A E, C D, A B, E D$ e $B C$, respectivamente.

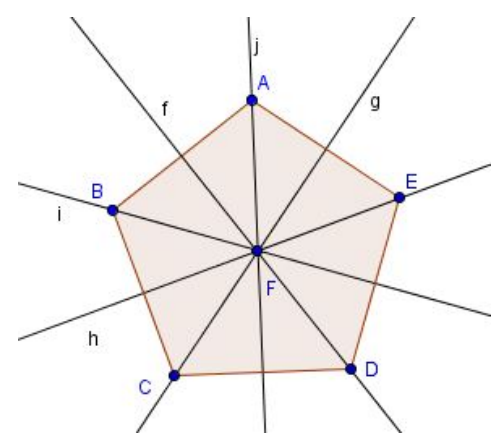

Figura 26: 
Os movimentos de reflexão em torno dos eixos de simetria axial são:

$$
\begin{gathered}
r_{g}=\left[\begin{array}{lllll}
A & B & C & D & E \\
E & D & C & B & A
\end{array}\right] r_{f}=\left[\begin{array}{lllll}
A & B & C & D & E \\
B & A & E & D & C
\end{array}\right] \\
r_{j}=\left[\begin{array}{lllll}
A & B & C & D & E \\
A & E & D & C & B
\end{array}\right] \\
r_{i}=\left[\begin{array}{lllll}
A & B & C & D & E \\
C & B & A & E & D
\end{array}\right] r_{h}=\left[\begin{array}{lllll}
A & B & C & D & E \\
D & C & B & A & E
\end{array}\right]
\end{gathered}
$$

Se rotacionarmos $0^{\circ}, 72^{\circ}, 144^{\circ}, 216^{\circ}$ e $288^{\circ}$ em torno de $F$ (centro do pentágono) no sentido anti-horário, teremos

$$
\begin{gathered}
I=\left[\begin{array}{lllll}
A & B & C & D & E \\
A & B & C & D & E
\end{array}\right] R_{1}=\left[\begin{array}{lllll}
A & B & C & D & E \\
E & A & B & C & D
\end{array}\right] \\
R_{2}=\left[\begin{array}{lllll}
A & B & C & D & E \\
D & E & A & B & C
\end{array}\right] \\
R_{3}=\left[\begin{array}{lllll}
A & B & C & D & E \\
C & D & E & A & B
\end{array}\right] R_{4}=\left[\begin{array}{lllll}
A & B & C & D & E \\
B & C & D & E & A
\end{array}\right]
\end{gathered}
$$

Logo essas transformações formam o grupo de simetria do pentágono regular que denominaremos $D_{5}=\left\{I, a, a^{2}, a^{3}, a^{4}, b, a b, a^{2} b, a^{3} b, a^{4} b\right\}$, onde $a=R_{72^{\circ}}, a^{2}=R_{144^{\circ}}, a^{3}=R_{216^{\circ}}, a^{4}=R_{288^{\circ}}$ e $b$ é uma reflexão do pentágono. Analogamente a conclusão feita para $\mathbb{D}_{4}$ temos que $\mathbb{D}_{5} \subset \mathbb{P}_{5}$.

A composição das transformações estão representados na tabela abaixo;

\begin{tabular}{c|cccccccccc}
${ }^{\circ}$ & $I$ & $r_{g}$ & $r_{f}$ & $r_{j}$ & $r_{i}$ & $r_{h}$ & $R_{1}$ & $R_{2}$ & $R_{3}$ & $R_{4}$ \\
\hline$r_{g}$ & $r_{g}$ & $I$ & $R_{2}$ & $R_{1}$ & $R_{3}$ & $R_{4}$ & $r_{j}$ & $r_{f}$ & $r_{i}$ & $r_{h}$ \\
$r_{f}$ & $r_{f}$ & $R_{3}$ & $I$ & $R_{4}$ & $R_{1}$ & $R_{2}$ & $r_{i}$ & $r_{h}$ & $r_{g}$ & $r_{j}$ \\
$r_{j}$ & $r_{j}$ & $R_{4}$ & $R_{1}$ & $I$ & $R_{2}$ & $R_{3}$ & $r_{f}$ & $r_{i}$ & $r_{h}$ & $r_{g}$ \\
$r_{i}$ & $r_{i}$ & $R_{2}$ & $R_{4}$ & $R_{3}$ & $I$ & $R_{1}$ & $r_{h}$ & $r_{g}$ & $r_{j}$ & $r_{f}$ \\
$r_{h}$ & $r_{h}$ & $R_{1}$ & $R_{3}$ & $R_{2}$ & $R_{4}$ & $I$ & $r_{g}$ & $r_{j}$ & $r_{f}$ & $r_{i}$ \\
$R_{1}$ & $R_{1}$ & $r_{h}$ & $r_{j}$ & $r_{g}$ & $r_{f}$ & $r_{i}$ & $R_{2}$ & $R_{3}$ & $R_{4}$ & $I$ \\
$R_{2}$ & $R_{2}$ & $r_{i}$ & $r_{g}$ & $r_{h}$ & $r_{j}$ & $r_{f}$ & $R_{3}$ & $R_{4}$ & $I$ & $R_{1}$ \\
$R_{3}$ & $R_{3}$ & $r_{f}$ & $r_{h}$ & $r_{i}$ & $r_{g}$ & $r_{j}$ & $R_{4}$ & $I$ & $R_{1}$ & $R_{2}$ \\
$R_{4}$ & $R_{4}$ & $r_{j}$ & $r_{i}$ & $r_{f}$ & $r_{h}$ & $r_{g}$ & $I$ & $R_{1}$ & $R_{2}$ & $R_{3}$
\end{tabular}




\subsection{Grupo de Simetrias de um Polígono Regular de $n$ lados}

Dado um polígono regular de $n$ lados centrado na origem do plano $\mathbb{R}^{2}$ com as mediatrizes de todos os lados, obteremos

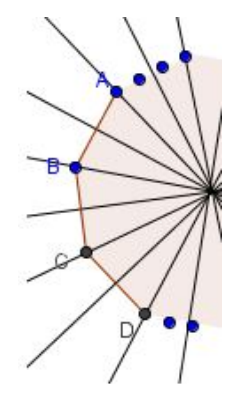

Figura 27:

Um grupo constituído por $n$ rotações de

$$
\frac{2 k \pi}{n}
$$

radianos $(k=0,1,2, \ldots n-1)$ em torno do centro do polígono e por $n$ reflexões nos eixos de simetrias do polígono.

Se o polígono tiver um número par de lados representado por $n=2 k$ teremos $k$ eixos de simetria passando por dois a dois vértices opostos e $k$ eixos passando pelos pontos médios de dois a dois lados opostos do polígono totalizando $2 k=n$ reflexões.

Se o polígono tiver um número ímpar de lados representado por $n=2 k+1$, teremos eixos de simetrias passando pelo ponto médio de cada lado e pelo vértice oposto a este lado, totalizando $2 k+1=n$ reflexões.

Assim, o grupo de simetrias de um polígono de $n$ lados é constituído por $2 n$ bijeções.

Ao refletirmos um polígono regular de $n$ lados em relação a uma reta $y=m x$ (eixo de simetria) em que $m=\operatorname{tg} \beta\left(\beta=0 \ldots 180^{\circ}\right)$, que passa pela origem do sistema cartesiano, teremos em forma matricial:

$$
r=\left[\begin{array}{cc}
\cos 2 \beta & \operatorname{sen} 2 \beta \\
\operatorname{sen} 2 \beta & -\cos 2 \beta
\end{array}\right]
$$

Ao rotacionarmos um polígono regular de $n$ lados em relação a origem sob um ângulo $\theta$, em sentido anti-horário, teremos: 


$$
R_{\theta}=\left[\begin{array}{cc}
\cos \theta & -\operatorname{sen} \theta \\
\operatorname{sen} \theta & \cos \theta
\end{array}\right]
$$

E assim fica fácil verificar que $I \in D_{n}$, já que:

$$
\left[\begin{array}{cc}
\cos \theta & -\operatorname{sen} \theta \\
\operatorname{sen} \theta & \cos \theta
\end{array}\right]\left[\begin{array}{cc}
\cos 0 & -\operatorname{sen} 0 \\
\operatorname{sen} 0 & \cos 0
\end{array}\right]=\left[\begin{array}{cc}
\cos \theta & -\operatorname{sen} \theta \\
\operatorname{sen} \theta & \cos \theta
\end{array}\right]
$$

e

$$
\left[\begin{array}{cc}
\cos 2 \beta & \operatorname{sen} 2 \beta \\
\operatorname{sen} 2 \beta & -\cos 2 \beta
\end{array}\right]\left[\begin{array}{cc}
\cos 0 & -\operatorname{sen} 0 \\
\operatorname{sen} 0 & \cos 0
\end{array}\right]=\left[\begin{array}{cc}
\cos 2 \beta & \operatorname{sen} 2 \beta \\
\operatorname{sen} 2 \beta & -\cos 2 \beta
\end{array}\right]
$$

onde

$$
I=\left[\begin{array}{cc}
\cos 0 & -\operatorname{sen} 0 \\
\operatorname{sen} 0 & \cos 0
\end{array}\right]=\left[\begin{array}{ll}
1 & 0 \\
0 & 1
\end{array}\right]
$$

De forma análoga o elemento inverso pertence a $D_{n}$

$$
\left[\begin{array}{cc}
\cos \theta & -\operatorname{sen} \theta \\
\operatorname{sen} \theta & \cos \theta
\end{array}\right]\left[\begin{array}{cc}
\cos \theta & \operatorname{sen} \theta \\
-\operatorname{sen} \theta & \cos \theta
\end{array}\right]=\left[\begin{array}{ll}
1 & 0 \\
0 & 1
\end{array}\right]
$$

onde

$$
R_{\theta}^{-1}=\left[\begin{array}{cc}
\cos \theta & \operatorname{sen} \theta \\
-\operatorname{sen} \theta & \cos \theta
\end{array}\right]
$$

é a inversa de $R_{\theta}$ sob uma rotação de um ângulo $\theta$ no sentido horário, e

$$
\left[\begin{array}{cc}
\cos 2 \beta & \operatorname{sen} 2 \beta \\
\operatorname{sen} 2 \beta & -\cos 2 \beta
\end{array}\right]\left[\begin{array}{cc}
\cos 2 \beta & \operatorname{sen} 2 \beta \\
\operatorname{sen} 2 \beta & -\cos 2 \beta
\end{array}\right]=\left[\begin{array}{ll}
1 & 0 \\
0 & 1
\end{array}\right]
$$

Logo a inversa da reflexão $r$ é dada por:

$$
r^{-1}=\left[\begin{array}{cc}
\cos 2 \beta & \operatorname{sen} 2 \beta \\
\operatorname{sen} 2 \beta & -\cos 2 \beta
\end{array}\right]
$$

Da composição de transformações temos:

$$
\begin{aligned}
& \left(\left[\begin{array}{cc}
\cos 2 \beta & \operatorname{sen} 2 \beta \\
\operatorname{sen} 2 \beta & -\cos 2 \beta
\end{array}\right]\left[\begin{array}{cc}
\cos \theta & -\operatorname{sen} \theta \\
\operatorname{sen} \theta & \cos \theta
\end{array}\right]\right)\left[\begin{array}{cc}
\cos k & \operatorname{senk} \theta \\
\operatorname{sen} \theta & -\operatorname{cosk} \theta
\end{array}\right]= \\
& {\left[\begin{array}{cc}
\cos 2 \beta & \operatorname{sen} 2 \beta \\
\operatorname{sen} 2 \beta & -\cos 2 \beta
\end{array}\right]\left(\left[\begin{array}{cc}
\cos \theta & -\operatorname{sen} \theta \\
\operatorname{sen} \theta & \cos \theta
\end{array}\right]\left[\begin{array}{cc}
\cos k & \operatorname{senk} \theta \\
\operatorname{sen} k & -\operatorname{cosk} \theta
\end{array}\right]\right)}
\end{aligned}
$$


De modo geral pela propriedade associativa de multiplicação de matrizes, dadas as matrizes $\mathrm{A}, \mathrm{B}$ e $\mathrm{C}$, temos:

$$
\begin{aligned}
& \mathrm{A}(\mathrm{BC})=\sum_{k=1}^{p} a_{i k} \sum_{l=1}^{q} b_{k l} c_{l j}=\sum_{k=1}^{p} \sum_{l=1}^{q} a_{i k} b_{k l} c_{l j}=\sum_{k=1}^{p} a_{i k} b_{k l} \sum_{l=1}^{q} c_{l j} \\
= & (\mathrm{AB}) \mathrm{C}
\end{aligned}
$$

Então propriedade associativa também é satisfeita, logo $D_{n}$ é um grupo. Esse grupo pode ser representado por $\mathbb{D}_{n}=\left\{I, a, a^{2}, a^{3}, \ldots, a^{n-1}, b, a b, a^{2} b\right.$, $\left.a^{3} b, \ldots, a^{n-1} b\right\}$, onde $n$ é o número de lados do polígono, $b=r$ (uma das reflexões do polígono), $a^{k}=R_{\theta_{k}}$ (rotações do polígono onde $R_{\theta_{0}}=I=R_{\theta_{n}}$ ) para $\theta_{k}=\frac{2 k \pi}{n}$ e $k=0,1, . ., n-1$.

Analisaremos, agora, a simetria de alguns poliedros.

\section{Grupo de Simetrias de Poliedros}

\subsection{Grupo de Simetrias dos Prismas}

Prisma é um sólido geométrico delimitado por faces planas, no qual as bases se situam em planos paralelos. Quanto a inclinação das arestas laterais, os prismas podem ser retos ou oblíquos.

\subsubsection{Grupo de Simetrias do Paralelepípedo}

O paralelepípedo é um prisma cuja base é um paralelogramo.

Considere o paralelepípedo com faces retangulares não quadradas e centro na origem do sistema cartesiano:

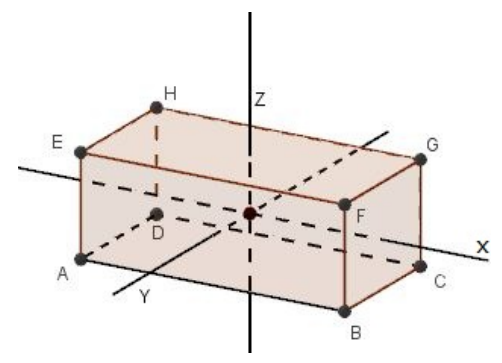

Figura 28:

Observe que ao rotacionarmos o paralelepípedo $180^{\circ}$, em sentido antihorário em torno de cada eixo coordenado, teremos 3 eixos de grau 2 que passam pelos centros das faces opostas coincidindo com os eixos $x, y$ e $z$ uma vez que o centro do paralelepípedo é a origem dos sistema cartesiano. Essas rotações estão descritas abaixo:

Em torno do eixo $x$ 


$$
R_{x}=\left[\begin{array}{llllllll}
A & B & C & D & E & F & G & H \\
H & G & F & E & D & C & B & A
\end{array}\right]
$$

Em torno do eixo $y$

$$
R_{y}=\left[\begin{array}{llllllll}
A & B & C & D & E & F & G & H \\
F & E & H & G & B & A & D & C
\end{array}\right]
$$

Em torno do eixo $z$

$$
R_{z}=\left[\begin{array}{llllllll}
A & B & C & D & E & F & G & H \\
C & D & A & B & G & H & E & F
\end{array}\right]
$$

A transformação identidade é dada por:

$$
I=\left[\begin{array}{llllllll}
A & B & C & D & E & F & G & H \\
A & B & C & D & E & F & G & H
\end{array}\right]
$$

Assim, o paralelepípedo possui, juntamente com a identidade, 4 rotações.

$$
\begin{aligned}
r_{x y} & =\left[\begin{array}{llllllll}
A & B & C & D & E & F & G & H \\
E & F & G & H & A & B & C & D
\end{array}\right] \\
r_{x z} & =\left[\begin{array}{llllllll}
A & B & C & D & E & F & G & H \\
D & C & B & A & H & G & F & E
\end{array}\right] \\
r_{y z} & =\left[\begin{array}{llllllll}
A & B & C & D & E & F & G & H \\
B & A & D & C & F & E & H & G
\end{array}\right] \\
r & =\left[\begin{array}{llllllll}
A & B & C & D & E & F & G & H \\
G & H & E & F & C & D & A & B
\end{array}\right]
\end{aligned}
$$

Logo o grupo de simetrias do paralelepípedo possui 8 transformações sendo $\mathbb{D}_{p}=\left\{I, R_{x}, R_{y}, R_{z}, r_{x y}, r_{x z}, r_{y z}, r\right\}$.

A composição entre as transformações estão descritas na tabela abaixo:

\begin{tabular}{c|cccccccc}
$\circ$ & $I$ & $R_{x}$ & $R_{y}$ & $R_{z}$ & $r_{x y}$ & $r_{x z}$ & $r_{y z}$ & $\mathrm{r}$ \\
\hline$I$ & $I$ & $R_{x}$ & $R_{y}$ & $R_{z}$ & $r_{x y}$ & $r_{x z}$ & $r_{y z}$ & $\mathrm{r}$ \\
$R_{x}$ & $R_{x}$ & $I$ & $R_{z}$ & $R_{y}$ & $r_{x z}$ & $r_{x y}$ & $r$ & $r_{y z}$ \\
$R_{y}$ & $R_{y}$ & $R_{z}$ & $I$ & $R_{x}$ & $r_{y z}$ & $r$ & $r_{x y}$ & $r_{x z}$ \\
$R_{z}$ & $R_{z}$ & $R_{y}$ & $R_{x}$ & $I$ & $r$ & $r_{y z}$ & $r_{x z}$ & $r_{x y}$ \\
$r_{x y}$ & $r_{x y}$ & $r_{x z}$ & $r_{y z}$ & $r$ & $I$ & $R_{x}$ & $R_{y}$ & $R_{z}$ \\
$r_{x z}$ & $r_{x z}$ & $r_{x y}$ & $r$ & $r_{y z}$ & $R_{x}$ & $I$ & $r_{y z}$ & $R_{y}$ \\
$r_{y z}$ & $r_{y z}$ & $r$ & $r_{x y}$ & $r_{x z}$ & $R_{y}$ & $R_{z}$ & $I$ & $R_{x}$ \\
$r$ & $r$ & $r_{x y}$ & $r_{x z}$ & $r_{x y}$ & $R_{z}$ & $R_{y}$ & $R_{x}$ & $I$
\end{tabular}


Observando a tabela verificamos que a composição de duas reflexões gera uma rotação e a composição das rotações com uma reflexão gera as outras reflexões. Assim, o grupo de simetrias para poliedros também pode ser descrito como:

$\mathbb{S}_{\text {paral }}=\left\{I, a, a_{2}, a_{3}, b, a b, a_{2} b, a_{3} b\right\}$ onde $a, a_{2}, a_{3}$ são as rotações em torno dos eixos coordenados, e $b=r_{x y}$ é uma reflexão em relação ao plano $x y$.

\subsubsection{Grupo de Simetrias do Prisma Pentagonal Reto}

Considere um prisma pentagonal regular reto com faces laterais retangulares não quadradas e com centro na origem do espaço cartesiano.

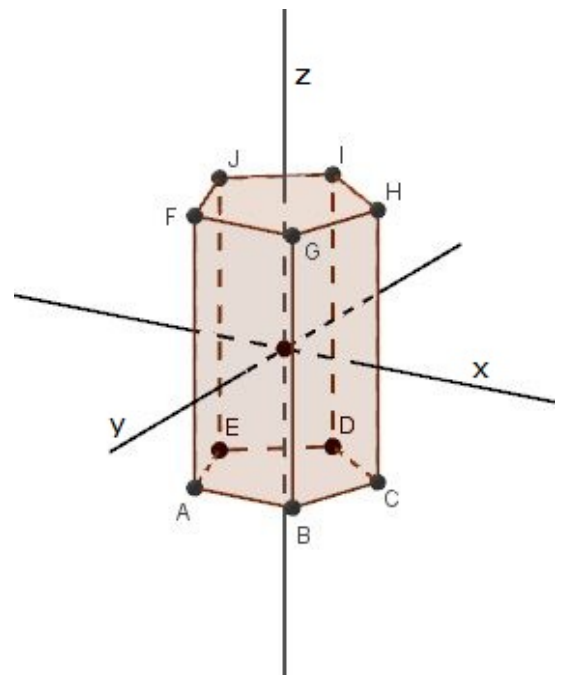

Figura 29:

No prisma pentagonal tem-se 1 eixo de grau 5 (eixo z) e 5 eixos de grau 2 que passa pela origem, pelo centro de cada retângulo e cruza o ponto médio de cada aresta vertical. As rotações em torno do eixo de grau 5 com giro anti-horário de $72^{\circ}, 144^{\circ}, 216^{\circ}, 288^{\circ}$ são descritas como:

$$
\begin{aligned}
R_{72^{\circ}} & =\left[\begin{array}{llllllllll}
A & B & C & D & E & F & G & H & I & J \\
E & A & B & C & D & J & F & G & H & I
\end{array}\right] \\
R_{144^{\circ}} & =\left[\begin{array}{llllllllll}
A & B & C & D & E & F & G & H & I & J \\
D & E & A & B & C & I & J & F & G & H
\end{array}\right] \\
R_{216^{\circ}} & =\left[\begin{array}{llllllllll}
A & B & C & D & E & F & G & H & I & J \\
C & D & E & A & B & H & I & J & F & G
\end{array}\right]
\end{aligned}
$$




$$
R_{288^{\circ}}=\left[\begin{array}{cccccccccc}
A & B & C & D & E & F & G & H & I & J \\
B & C & D & E & A & G & H & I & J & F
\end{array}\right]
$$

Além disso há 5 eixos de grau 2 que passam pelo ponto médio das arestas laterais e pelo centro da face oposta a aresta. As possíveis rotações em torno de cada um desses eixos no sentido anti-horário num ângulo de $180^{\circ}$ estão descritas nos esquemas abaixo:

$$
\begin{aligned}
R_{G B} & =\left[\begin{array}{llllllllll}
A & B & C & D & E & F & G & H & I & J \\
H & G & F & J & I & C & B & A & E & D
\end{array}\right] \\
R_{H C} & =\left[\begin{array}{llllllllll}
A & B & C & D & E & F & G & H & I & J \\
J & I & H & G & F & E & D & C & B & A
\end{array}\right] \\
R_{I D} & =\left[\begin{array}{lllllllllll}
A & B & C & D & E & F & G & H & I & J \\
G & F & H & J & I & B & A & E & E & D
\end{array}\right] \\
R_{J E} & =\left[\begin{array}{lllllllllll}
A & B & C & D & E & F & G & H & I & J \\
I & H & G & F & J & D & C & B & A & E
\end{array}\right] \\
R_{F A} & =\left[\begin{array}{llllllllll}
A & B & C & D & E & F & G & H & I & J \\
F & J & I & H & G & A & E & D & C & B
\end{array}\right]
\end{aligned}
$$

Assim, o número de rotações é dado por:

$$
1.4+5.1=9
$$

Logo o número de rotações mais a identidade perfazem um total de 10 .

Sabendo que a partir da composição de cada rotação com uma reflexão são obtidas as outras reflexões, tomemos a reflexão obtida através do plano que passa pelos pontos médios das arestas laterais, e assim:

$$
r_{x y}=\left[\begin{array}{llllllllll}
A & B & C & D & E & F & G & H & I & J \\
F & G & H & I & J & A & B & C & D & E
\end{array}\right]
$$

$R_{72^{\circ}} r_{x y}$

$$
r_{1}=\left[\begin{array}{llllllllll}
A & B & C & D & E & F & G & H & I & J \\
J & F & G & H & I & E & A & B & C & D
\end{array}\right]
$$




$$
R_{144^{\circ}} r_{x y}
$$

$$
r_{2}=\left[\begin{array}{cccccccccc}
A & B & C & D & E & F & G & H & I & J \\
I & J & F & G & H & D & E & A & B & C
\end{array}\right]
$$

$R_{216^{\circ}} r_{x y}$

$$
r_{3}=\left[\begin{array}{llllllllll}
A & B & C & D & E & F & G & H & I & J \\
H & I & J & F & G & C & D & E & A & B
\end{array}\right]
$$

$R_{288^{\circ}} r_{x y}$

$$
r_{4}=\left[\begin{array}{llllllllll}
A & B & C & D & E & F & G & H & I & J \\
G & H & I & J & F & B & C & D & I & A
\end{array}\right]
$$

$R_{G B} r_{x y}$

$$
r_{G B}=\left[\begin{array}{llllllllll}
A & B & C & D & E & F & G & H & I & J \\
C & B & A & E & D & H & G & F & J & I
\end{array}\right]
$$

$R_{H C} r_{x y}$

$$
r_{H C}=\left[\begin{array}{llllllllll}
A & B & C & D & E & F & G & H & I & J \\
E & D & C & B & A & J & I & H & G & F
\end{array}\right]
$$

$R_{I D} r_{x y}$

$$
r_{I D}=\left[\begin{array}{cccccccccc}
A & B & C & D & E & F & G & H & I & J \\
B & A & E & D & C & G & F & J & I & H
\end{array}\right]
$$

$R_{J E} r_{x y}$

$$
r_{J E}=\left[\begin{array}{llllllllll}
A & B & C & D & E & F & G & H & I & J \\
D & C & B & A & E & I & H & G & F & J
\end{array}\right]
$$

$R_{F A} r_{x y}$

$$
r_{F A}=\left[\begin{array}{cccccccccc}
A & B & C & D & E & F & G & H & I & J \\
A & E & D & C & B & F & J & I & H & G
\end{array}\right]
$$

Logo o grupo de simetrias do prisma pentagonal reto de faces laterais retangulares (não quadrada) é descrito pelo conjunto:

$\mathbb{S}_{\text {prismapent }}=\left\{I, a, a^{2}, a^{3}, a^{4}, b, a b, a^{2} b, a^{3} b, a^{4} b, a_{1}, a_{1} b, a_{2}, a_{2} b, a_{3}, a_{3} b, a_{4}\right.$, $\left.a_{4} b, a_{5}, a_{5} b\right\}$ onde $a, a^{2}, a^{3}, a^{4}$ são as rotações em torno do eixo $z$ de grau $5 \mathrm{e}$ $a_{1}, a_{2}, a_{3}, a_{4}$ e $a_{5}$ são as rotações em torno dos eixos de grau 2 que passam pela origem, cruzam o ponto médio de cada aresta vertical e passam pelo centro das faces laterais.

Assim o prisma pentagonal possui 20 simetrias. 


\subsubsection{Grupo de Simetrias de um Prisma de $n$ faces laterais}

Considere o prisma cuja base é um polígono de $n$ lados com faces retangulares não quadradas e centro na origem do sistema cartesiano:

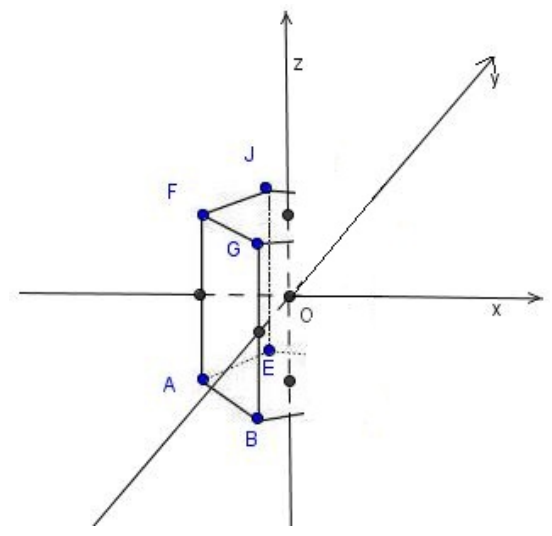

Figura 30:

$\mathbb{S}_{\text {prisman }}=\left\{I, a_{1}, \ldots, a_{n}, a, a^{2}, \ldots, a^{n-1}, b, a b, a_{1} b, \ldots, a_{n} b, a^{2} b, \ldots, a^{n-1} b\right\}$ onde $a_{1}, \ldots, a_{n}$ são as rotações em torno dos eixos de grau $2 ; a, a^{2}, \ldots, a^{n-1}$ são as rotações em torno do eixo $z$ de grau $n$ e $b=r_{x y}$ é a reflexão em relação ao plano $x y$.

Se o polígono da base do prisma tiver um número par de lados, os eixos de grau 2 passam pela origem e pelos pontos médios das arestas verticais opostas e outros que passam pelos centros das faces opostas.

Se o polígono da base do prisma tiver um número ímpar de lados, os eixos de grau 2 passam pela origem, pelo ponto médio da aresta vertical e pelo centro da face oposta.

Seja $p$ o número de eixos de simetria do polígono da base, então o grupo de simetrias de um prisma (nestas condições) é composto por $4 p$ transformações.

\subsection{Grupo de Simetrias das Pirâmides}

Consideremos um polígono convexo situado num plano e um ponto $V$ fora deste plano. Chama-se pirâmide a reunião dos segmentos com uma extremidade em $V$ e a outra nos pontos do polígono.

\subsubsection{Grupo de Simetrias da Pirâmide Hexagonal Reta}

Consideremos a pirâmide reta com base um hexágono regular cujas laterais são triângulos isósceles. O hexágono está no plano $x y$ com centro no ponto de coordenadas $(0,0,0)$ e o vértice está no eixo $z$ (positivo). 


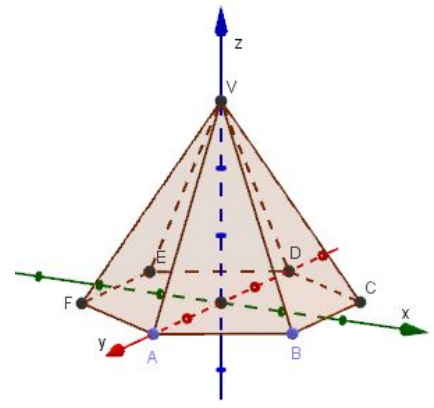

Figura 31:

A pirâmide apresenta seis rotações em torno do eixo $z$, apresentadas como:

$$
I=\left[\begin{array}{lllllll}
A & B & C & D & E & F & G \\
A & B & C & D & E & F & G
\end{array}\right] \quad R_{60^{\circ}}=\left[\begin{array}{ccccccc}
A & B & C & D & E & F & G \\
F & A & B & C & D & E & G
\end{array}\right]
$$

$R_{120^{\circ}}=\left[\begin{array}{ccccccc}A & B & C & D & E & F & G \\ E & F & A & B & C & D & G\end{array}\right] \quad R_{180^{\circ}}=\left[\begin{array}{ccccccc}A & B & C & D & E & F & G \\ D & E & F & A & B & C & G\end{array}\right]$

$R_{240^{\circ}}=\left[\begin{array}{lllllll}A & B & C & D & E & F & G \\ C & D & E & F & A & B & G\end{array}\right] \quad R_{300^{\circ}}=\left[\begin{array}{ccccccc}A & B & C & D & E & F & G \\ B & C & D & E & F & A & G\end{array}\right]$

Considerando os planos de simetria formados pelo ponto $G$ e pelas retas que passam pelos vértices da base da pirâmide (denominados por $B E, A D$ e $C F$ ) ou pelas retas que passam pelo pontos médios dos lados do polígono da base (denominados por $A B, B C$ e $C D$ ), teremos as reflexões:

$$
\begin{aligned}
& r_{B E}=\left[\begin{array}{lllllll}
A & B & C & D & E & F & G \\
C & B & A & F & E & D & G
\end{array}\right] r_{A D}=\left[\begin{array}{lllllll}
A & B & C & D & E & F & G \\
A & F & E & D & C & B & G
\end{array}\right] \\
& r_{C F}=\left[\begin{array}{lllllll}
A & B & C & D & E & F & G \\
E & D & C & B & A & F & G
\end{array}\right] r_{A B}=\left[\begin{array}{lllllll}
A & B & C & D & E & F & G \\
B & A & F & E & D & C & G
\end{array}\right] \\
& r_{B C}=\left[\begin{array}{llllllll}
A & B & C & D & E & F & G \\
D & C & B & A & F & E & G
\end{array}\right] \quad r_{C D}=\left[\begin{array}{lllllll}
A & B & C & D & E & F & G \\
F & E & D & C & B & A & G
\end{array}\right]
\end{aligned}
$$

Logo o grupo de simetrias da pirâmide reta hexagonal possui 12 transformações sendo descrito por:

$\mathbb{S}_{\text {piran }}=\left\{I, a, a^{2}, a^{3}, a^{4}, a^{5}, b, a b, a^{2} b, a^{3} b, a^{4} b, a^{5} b\right\}$ onde $a, a^{2}, a^{3} \cdot a^{4}, a^{5}$ são as rotações em torno do eixo $z$ (grau 6 ) e $b=r$ é uma das reflexões da pirâmide. 


\subsubsection{Grupo de Simetrias da Pirâmide Reta de $n$ faces laterais}

Considerando a pirâmide reta cuja base é um polígono de $n$ lados e as faces laterais são triângulos isósceles e centro na origem do sistema cartesiano:

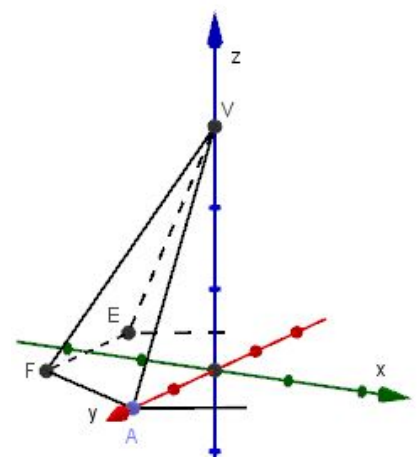

Figura 32:

$\mathbb{S}_{\text {piran }}=\left\{I, a, a^{2}, \ldots, a^{n-1}, b, a b, a^{2} b, \ldots, a^{n-1} b\right\}$ onde $a, a^{2}, \ldots, a^{n-1}$ são $\quad$ as rotações de em torno do eixo $z$ de grau $n$ e $b=r$ é uma reflexão em relação ao plano que passa pelo eixo $z$ e pelos pontos médios das arestas opostas ou pelos vértices opostos do polígono da base quando o número de lados for par. Se o número de lados do polígono da base for ímpar a reflexão é em relação ao plano que passa pelo eixo $z$, pelo ponto médio da aresta e pelo vértice oposto.

Assim com a composição das $n-1$ rotações com uma reflexão teremos mais $n-1$ reflexões perfazendo, juntamente com a identidade, $2 n$ simetrias.

Podemos assim concluir que sendo $p$ o número de eixos de simetria do polígono da base então o grupo de simetrias da pirâmide é composto por $2 p$ transformações.

Sabendo que as rotações e as reflexões podem ser representadas matricialmente, então fica trivial demonstrarmos que o conjunto das rotações e reflexões de um poliedro é um grupo.

A matriz Identidade pertence ao grupo de simetrias do poliedro já que:

$$
I=\left[\begin{array}{ccc}
\cos 0 & -\operatorname{sen} 0 & 0 \\
\operatorname{sen} 0 & \cos 0 & 0 \\
0 & 0 & 1
\end{array}\right]=\left[\begin{array}{lll}
1 & 0 & 0 \\
0 & 1 & 0 \\
0 & 0 & 1
\end{array}\right]
$$

Cada elemento do grupo possui um inverso pertencente a esse grupo.

$$
R_{x} R_{x}^{-1}=\left[\begin{array}{ccc}
1 & 0 & 0 \\
0 & \cos \theta & -\operatorname{sen} \theta \\
0 & \operatorname{sen} \theta & \cos \theta
\end{array}\right]\left[\begin{array}{ccc}
1 & 0 & 0 \\
0 & \cos \theta & \operatorname{sen} \theta \\
0 & -\operatorname{sen} \theta & \cos \theta
\end{array}\right]=\left[\begin{array}{lll}
1 & 0 & 0 \\
0 & 1 & 0 \\
0 & 0 & 1
\end{array}\right]
$$




$$
\begin{gathered}
R_{y} R_{y}^{-1}=\left[\begin{array}{ccc}
\cos \theta & 0 & -\operatorname{sen} \theta \\
0 & 1 & 0 \\
\operatorname{sen} \theta & 0 & \cos \theta
\end{array}\right]\left[\begin{array}{ccc}
\cos \theta & 0 & \operatorname{sen} \theta \\
0 & 1 & 0 \\
-\operatorname{sen} \theta & 0 & \cos \theta
\end{array}\right]=\left[\begin{array}{lll}
1 & 0 & 0 \\
0 & 1 & 0 \\
0 & 0 & 1
\end{array}\right] \\
R_{z} R_{z}^{-1}=\left[\begin{array}{ccc}
\cos \theta & -\operatorname{sen} \theta & 0 \\
\operatorname{sen} \theta & \cos \theta & 0 \\
0 & 0 & 1
\end{array}\right]\left[\begin{array}{ccc}
\cos \theta & \operatorname{sen} \theta & 0 \\
-\operatorname{sen} \theta & \cos \theta & 0 \\
0 & 0 & 1
\end{array}\right]=\left[\begin{array}{lll}
1 & 0 & 0 \\
0 & 1 & 0 \\
0 & 0 & 1
\end{array}\right] \\
r_{x m} r_{x m}^{-1}=\left[\begin{array}{ccc}
1 & 0 & 0 \\
0 & \cos 2 \beta & \operatorname{sen} 2 \beta \\
0 & \operatorname{sen} 2 \beta & -\cos 2 \beta
\end{array}\right]\left[\begin{array}{ccc}
1 & 0 & 0 \\
0 & \cos 2 \beta & \operatorname{sen} 2 \beta \\
0 & \operatorname{sen} 2 \beta & -\cos 2 \beta
\end{array}\right]=\left[\begin{array}{lll}
1 & 0 & 0 \\
0 & 1 & 0 \\
0 & 0 & 1
\end{array}\right] \\
r_{y m} r_{y m}^{-1}=\left[\begin{array}{ccc}
\cos 2 \beta & 0 & \operatorname{sen} 2 \beta \\
0 & 1 & 0 \\
\operatorname{sen} 2 \beta & 0 & -\cos 2 \beta
\end{array}\right]\left[\begin{array}{ccc}
\cos 2 \beta & 0 & \operatorname{sen} 2 \beta \\
0 & 1 & 0 \\
\operatorname{sen} 2 \beta & 0 & -\cos 2 \beta
\end{array}\right]=\left[\begin{array}{lll}
1 & 0 & 0 \\
0 & 1 & 0 \\
0 & 0 & 1
\end{array}\right] \\
r_{z m} r_{z m}^{-1}=\left[\begin{array}{ccc}
\cos 2 \beta & \operatorname{sen} 2 \beta & 0 \\
\operatorname{sen} 2 \beta & -\cos 2 \beta & 0 \\
0 & 0 & 1
\end{array}\right]\left[\begin{array}{ccc}
\cos 2 \beta & \operatorname{sen} 2 \beta & 0 \\
\operatorname{sen} 2 \beta & -\cos 2 \beta & 0 \\
0 & 0 & 1
\end{array}\right]=\left[\begin{array}{lll}
1 & 0 & 0 \\
0 & 1 & 0 \\
0 & 0 & 1
\end{array}\right]
\end{gathered}
$$

E finalmente como a propriedade associativa é satisfeita para multiplicação de matrizes então vale para a composição de simetrias.

Assim, por exemplo:

$$
\begin{aligned}
& \left(r_{z m} R_{x}\right) r_{y m}= \\
& \left(\left[\begin{array}{ccc}
\cos 2 \beta & \operatorname{sen} 2 \beta & 0 \\
\operatorname{sen} 2 \beta & -\cos 2 \beta & 0 \\
0 & 0 & 1
\end{array}\right]\left[\begin{array}{ccc}
1 & 0 & 0 \\
0 & \cos \theta & -\operatorname{sen} \theta \\
0 & \operatorname{sen} \theta & \cos \theta
\end{array}\right]\right)\left[\begin{array}{ccc}
\cos 2 \beta & 0 & \operatorname{sen} 2 \beta \\
0 & 1 & 0 \\
\operatorname{sen} 2 \beta & 0 & -\cos 2 \beta
\end{array}\right] \\
= & {\left[\begin{array}{ccc}
\cos 2 \beta & \operatorname{sen} 2 \beta & 0 \\
\operatorname{sen} 2 \beta & -\cos 2 \beta & 0 \\
0 & 0 & 1
\end{array}\right]\left(\left[\begin{array}{ccc}
1 & 0 & 0 \\
0 & \cos \theta & -\operatorname{sen} \theta \\
0 & \operatorname{sen} \theta & \cos \theta
\end{array}\right]\left[\begin{array}{ccc}
\cos 2 \beta & 0 & \operatorname{sen} 2 \beta \\
0 & 1 & 0 \\
\operatorname{sen} 2 \beta & 0 & -\cos 2 \beta
\end{array}\right]\right) } \\
= & r_{z m}\left(R_{x} r_{y m}\right)
\end{aligned}
$$

\subsection{Grupo de Simetrias dos Poliedros Regulares}

Um poliedro convexo é chamado de regular se suas faces são polígonos regulares, cada um com o mesmo número de lados e, para todo vértice, converge um mesmo número de arestas.

Existem cinco poliedros regulares também chamados de Poliedros de Platão: Tetraedro, Hexaedro, Octaedro, Dodecaedro e Icosaedro. 


\subsubsection{Grupo de Simetrias do Tetraedro Regular}

O tetraedro regular é um poliedro regular formado por quatro triângulos equiláteros.

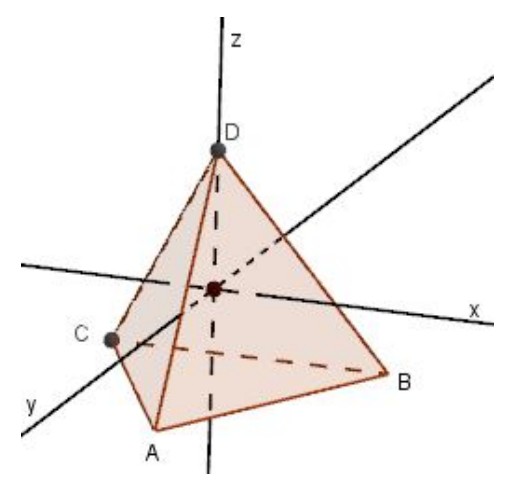

Figura 33:

Como o tetraedro possui 4 vértices então o grupo de simetrias é menor ou igual ao grupo de permutações desses 4 pontos, ou seja, o grupo de simetrias é menor ou igual a $24=4$ !.

Para analisarmos as simetrias do tetraedro tomaremos as rotações de $120^{\circ}$ e $240^{\circ}$, no sentido anti-horário, em torno dos eixos que passam pelo vértice e centro da face oposta, denominados eixos de grau 3, e assim obteremos:

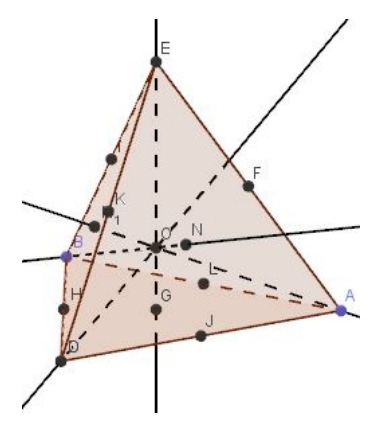

Figura 34:

$$
\begin{aligned}
& R_{D 120}=\left[\begin{array}{llll}
A & B & C & D \\
C & A & B & D
\end{array}\right] R_{D 240}=\left[\begin{array}{cccc}
A & B & C & D \\
B & C & A & D
\end{array}\right] \\
& R_{C 120}=\left[\begin{array}{llll}
A & B & C & D \\
A & D & C & A
\end{array}\right] R_{C 240}=\left[\begin{array}{llll}
A & B & C & D \\
D & A & C & B
\end{array}\right]
\end{aligned}
$$




$$
\begin{aligned}
& R_{B 120}=\left[\begin{array}{llll}
A & B & C & D \\
D & B & A & C
\end{array}\right] R_{B 240}=\left[\begin{array}{cccc}
A & B & C & D \\
C & B & D & A
\end{array}\right] \\
& R_{A 120}=\left[\begin{array}{llll}
A & B & C & D \\
A & C & D & B
\end{array}\right] R_{A 240}=\left[\begin{array}{llll}
A & B & C & D \\
A & D & B & C
\end{array}\right]
\end{aligned}
$$

Além dessas rotações temos a identidade e as em torno dos eixos que passam pelos pontos médios das arestas que não se intersectam, denominados eixos de grau 2, temos:

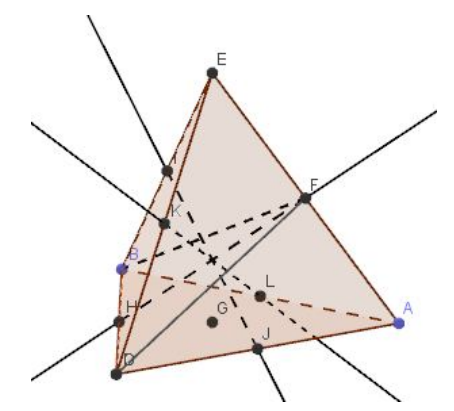

Figura 35:

$$
\begin{gathered}
I=\left[\begin{array}{cccc}
A & B & C & D \\
A & B & C & D
\end{array}\right] \quad R_{A B}=\left[\begin{array}{cccc}
A & B & C & D \\
B & A & D & C
\end{array}\right] \\
R_{B C}=\left[\begin{array}{llll}
A & B & C & D \\
D & C & B & A
\end{array}\right] \quad R_{A C}=\left[\begin{array}{cccc}
A & B & C & D \\
C & A & D & C
\end{array}\right]
\end{gathered}
$$

Como o tetraedro possui três eixos de grau 2 com giro de $180^{\circ}$ e quatro eixos de grau 3 com giro de $120^{\circ}$ e $240^{\circ}$, então

$$
3.1+4.2=11
$$

Incluindo a identidade tem-se 12 rotações.

$$
\begin{gathered}
r_{A B}=\left[\begin{array}{llll}
A & B & C & D \\
B & A & C & D
\end{array}\right] r_{A D}=\left[\begin{array}{cccc}
A & B & C & D \\
D & B & C & A C
\end{array}\right] \\
r_{B D}=\left[\begin{array}{cccc}
A & B & C & D \\
A & D & C & B
\end{array}\right] r_{A C}=\left[\begin{array}{llll}
A & B & C & D \\
C & B & A & D
\end{array}\right]
\end{gathered}
$$




$$
\begin{aligned}
r_{B C} & =\left[\begin{array}{llll}
A & B & C & D \\
A & C & B & D
\end{array}\right] r_{C D}=\left[\begin{array}{llll}
A & B & C & D \\
A & B & D & C
\end{array}\right] \\
R_{A 120} & =\left[\begin{array}{llll}
A & B & C & D \\
C & A & B & D
\end{array}\right] R_{A 240}=\left[\begin{array}{llll}
A & B & C & D \\
B & C & A & D
\end{array}\right]
\end{aligned}
$$

Além dessas rotações, o tetraedro possui a identidade com um giro de $360^{\circ}$ e outras três rotações de $180^{\circ}$ em torno dos eixos que passam pelos pontos médios das arestas que não se intersectam.

$$
\begin{aligned}
& R_{A 120}=\left[\begin{array}{llll}
A & B & C & D \\
C & A & B & D
\end{array}\right] \quad R_{A 240}=\left[\begin{array}{llll}
A & B & C & D \\
B & C & A & D
\end{array}\right] \\
& R_{A 120}=\left[\begin{array}{llll}
A & B & C & D \\
C & A & B & D
\end{array}\right] R_{A 240}=\left[\begin{array}{llll}
A & B & C & D \\
B & C & A & D
\end{array}\right]
\end{aligned}
$$

Denominando $a, a^{2}, a_{1}, a_{1}^{2}, a_{2}, a_{2}^{2}, a_{3}, a_{3}^{2}$ as rotações em torno dos eixos de grau $3, s_{1}, s_{2}$ e $s_{3}$ as rotações em torno dos eixos de grau 2 e $b$ uma reflexão, obteremos ao compor essa única reflexão com as rotações as outras reflexões, perfazendo 24 isometrias do tetraedro. Esse grupo de simetrias pode ser descrito por:

$$
\begin{aligned}
& \mathbb{D}_{t e t r a}=\left\{I, a, a^{2}, a_{1}, a_{1}^{2}, a_{2}, a_{2}^{2}, a_{3}, a_{3}^{2}, b, a b, a^{2} b, a_{1} b, a_{1}^{2} b, a_{2} b, a_{2}^{2} b, a_{3} b, a_{3}^{2} b, s_{1},\right. \\
& \left.s_{2}, s_{3}, s_{1} b, s_{2} b, s_{3} b\right\}
\end{aligned}
$$

\subsubsection{Grupo de Simetrias do Hexaedro Regular ou Cubo}

O hexaedro regular é um poliedro regular formado por seis quadrados. São identificados no cubo três tipos de eixos de rotação, onde 6 são de grau 2, 4 de grau 3 e 3 de grau 4.

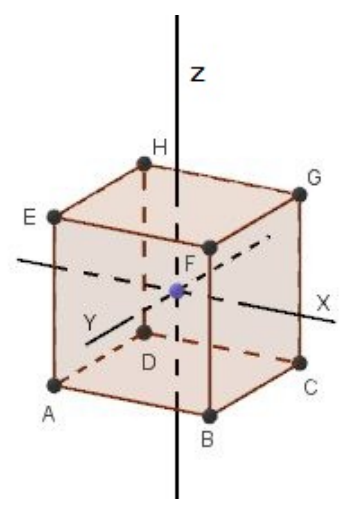

Figura 36: 
As rotações do cubo são obtidas com um giro de $180^{\circ}$ em torno dos eixos de grau 2 que passam pelos pontos médios de cada par de arestas opostas, com giro de $120^{\circ}$ e $240^{\circ}$ em torno dos eixos de grau 3 que passam por cada par de vértices opostos, e finalmente com giros de $90^{\circ}, 180^{\circ}$ e $270^{\circ}$ em torno dos eixos de grau 4 que passam pelo centro de cada par de faces opostas.

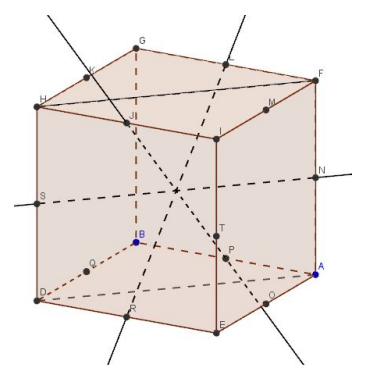

Figura 37:

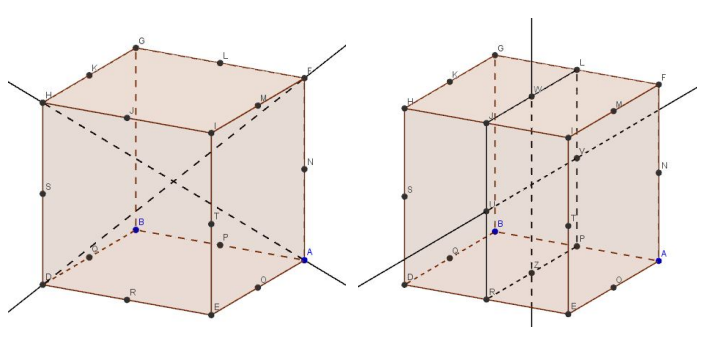

Figura 38:

Figura 39:

Assim teremos,

$$
6.1+4.2+3.3=23
$$

e incluindo a identidade 24 simetrias de rotação e como as composições de uma única reflexão com as rotações geram as outras reflexões então o grupo de simetrias do cubo possui 48 isometrias.

\subsubsection{Grupo de Simetrias do Octaedro Regular}

O octaedro regular é um poliedro regular formado por seis triângulos equiláteros.

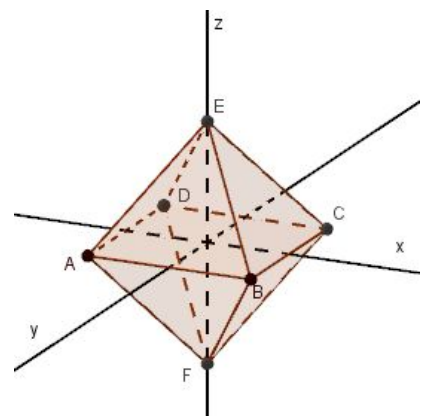

Figura 40:

O octaedro possui 6 eixos de grau 2, 4 eixos de grau 3 e 3 eixos de grau 4 . 


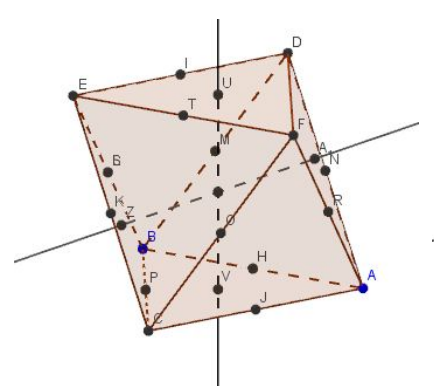

Figura 41:

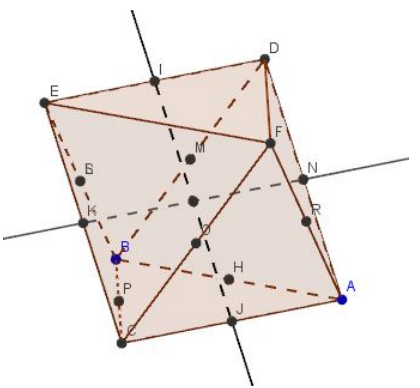

Figura 42:

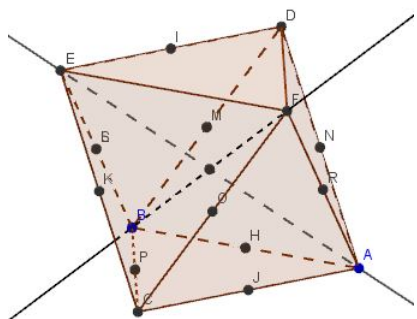

Figura 43:

O número de rotações do octaedro é igual ao do hexaedro. O grupo de simetrias de ambos são isomorfos, assim o grupo de simetrias do octaedro também é composto por 48 isometrias.

\subsubsection{Grupo de Simetrias do Dodecaedro Regular}

O dodecaedro regular é um poliedro regular formado por 12 pentágonos regulares.

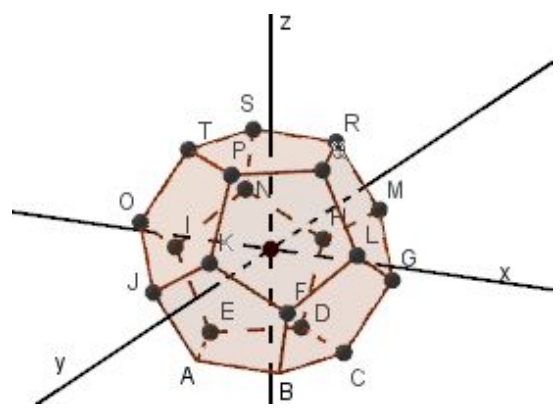

Figura 44:

Esse poliedro possui três tipos de eixos de rotação sendo 15 de grau 2, 10 de grau 3 e 6 de grau 5 .

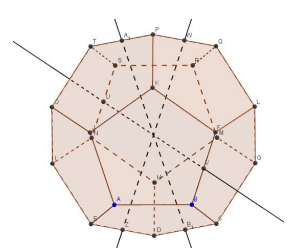

Figura 45:

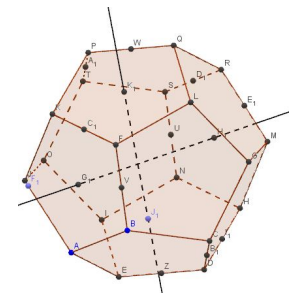

Figura 46:

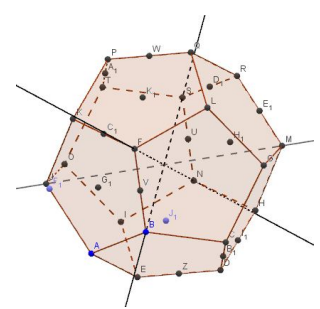

Figura 47: 
As rotações do dodecaedro são obtidas sob um giro de $180^{\circ}$ em torno dos eixos de grau 2 que passam pelos pontos médios de cada par de arestas opostas, com giro de $120^{\circ}$ e $240^{\circ}$ em torno dos eixos de grau 3 que passam por cada par de vértices opostos e com giros de $72^{\circ}, 144^{\circ}, 216^{\circ}$ e $288^{\circ}$ em torno dos eixos de grau 5 que passam pelo centro de cada par de faces opostas.

Assim

$$
15.1+10.2+6.4=59
$$

teremos, incluindo a identidade 60 simetrias de rotação e como cada composição de uma reflexão com as rotações geram as outras reflexões então o grupo de simetrias do dodecaedro possui 120 simetrias.

\subsubsection{Grupo de Simetrias do Icosaedro Regular}

O icosaedro regular é um poliedro regular formado por 20 triângulos equiláteros.

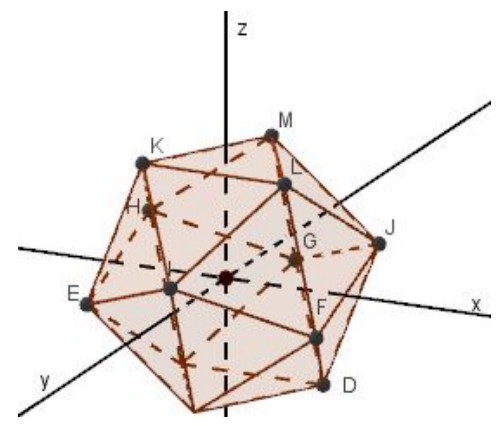

Figura 48:

Esse poliedro possui três tipos de eixos de rotação sendo 15 de grau 2, 10 de grau 3 e 6 de grau 5. O grupo de simetrias do icosaedro é isomorfo ao grupo de simetria do dodecaedro.

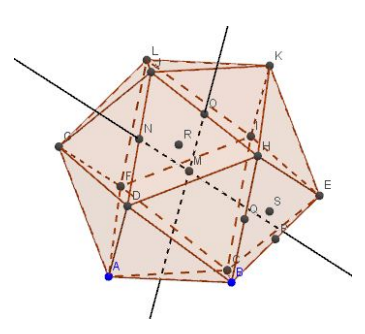

Figura 49:

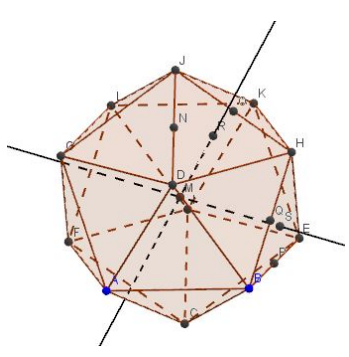

Figura 50:

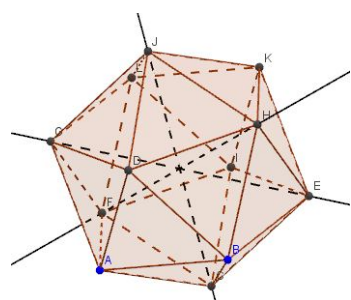

Figura 51:

Assim, o icosaedro possui 120 simetrias. 


\section{Aplicações}

Na Física, um sistema físico possui uma simetria quando após ser realizada uma operação sobre o sistema ele permanecer inalterado. O conceito de simetria é muito usado pelos físicos para descrição de muitos fenômenos como o das forças forte e fraca que interagem no núcleo atômico.

Entretanto se destaca a aplicação do conceito de simetria no conhecido teorema de Noether: "para cada simetria que encontramos em um sistema físico temos associada uma lei de conservação, ou seja, uma grandeza do sistema mantém seu valor (permanece inalterada) quando realizamos a operação associada a simetria". Devido a isso é que os físicos dão tanta importância a simetria pois é a conservação das grandezas que os permitem "prever a evolução dos sistemas", como se o Sol vai engolir os planetas, entre outros fenômenos da Natureza (Uma década em busca da simetria da natureza S.F. Novaes - IFT/Unesp).

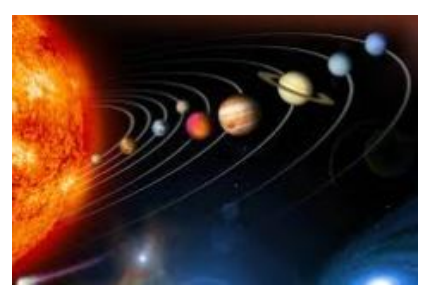

Figura 52:

Na Química o conceito de simetria ajuda a determinar propriedades físicas das moléculas, a construir orbitais moleculares além de indicar reações e gerar discussões em outros aspectos. Como exemplo de aplicação desse conceito, as moléculas que possuem pelo menos um dos elementos: plano, eixo e centro de simetria, são simétricas e, por isso, não têm atividade óptica (opticamente inativas), ou seja, não desviam o plano da luz polarizada. Já as moléculas que possuem somente o eixo de simetria podem apresentar atividade óptica e são ditas dissimétricas. No entanto as moléculas que não posuem eixo, plano ou centro de simetria são assimétricas e ditas opticamente ativas (Simetria Molecular - Prof. Augusto Leite Coelho - UECe).

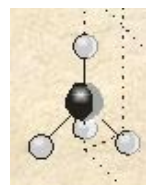

Figura 53: 
Analisando uma molécula de água verificaremos 4 simetrias : $I, R, r_{x z} \mathrm{e}$ $r_{y z}$.

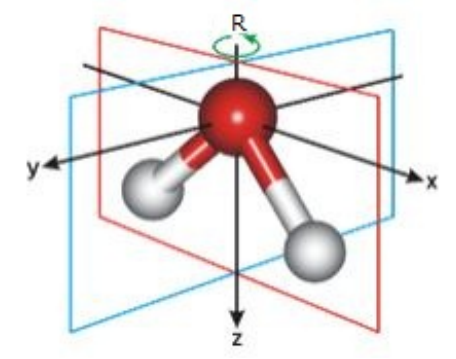

Figura 54:

\begin{tabular}{c|cccc}
$\circ$ & $I$ & $R$ & $r_{x z}$ & $r_{y z}$ \\
\hline$I$ & $I$ & $R$ & $r_{x z}$ & $r_{y z}$ \\
$R$ & $R$ & $I$ & $r_{y z}$ & $r_{x z}$ \\
$r_{x z}$ & $r_{x z}$ & $r_{y z}$ & $I$ & $R$ \\
$r_{y z}$ & $r_{y z}$ & $r_{x z}$ & $R$ & $I$
\end{tabular}

Através da tabela verificamos que as composições obedecem as propriedades de grupo e em particular é um grupo abeliano pois há comutatividade. Constatando as propriedades de simetria de uma molécula podemos definir o grupo de pontos a qual pertence. Temos por exemplo as moléculas pertencentes ao grupo de pontos $C_{1}(\mathrm{Si}, \mathrm{I}, \mathrm{Cl}, \mathrm{Br}, \mathrm{F}), \mathrm{C}_{2}\left(\mathrm{H}_{2} \mathrm{O}_{2}\right), \mathrm{C}_{5}\left(\mathrm{NHF}_{2}\right)$, entre outros perfazendo 32 grupos. A partir disso é possível dizer se a molécula é polar ou quiral, além de possiblitar a construção e classificação dos orbitais moleculares e a interpretação dos dados espectográficos usados na determinação das estruturas.

Na Biologia existe a distinção entre os grupos que possuem simetria radial e simetria bilateral. A simetria auxilia na coordenação da locomoção, enquanto os animais bilateralmente simétricos andam, nadam, rastejam ou pulam, os que possuem simetria radial, na maioria das vezes, são sésseis (não se locomovem) ou possuem locomoção ambulacrária, como é o caso dos equinodermatas. Sendo assim, a simetria afeta mais do que o visual e sim a sobrevivência e a adaptação a certos ambientes (bioajuda.blogspot.com.br). 

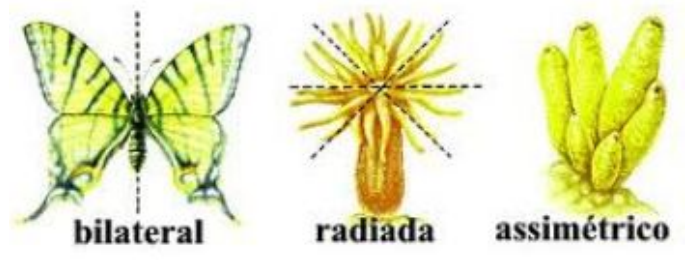

Figura 55:

$\mathrm{Na}$ Arte a simetria representa beleza, harmonia e perfeição então muitos adotaram e adotam esse conceito nas suas obras embora outros prefiram representar a assimetria como forma de expressar a criatividade e o inesperado.

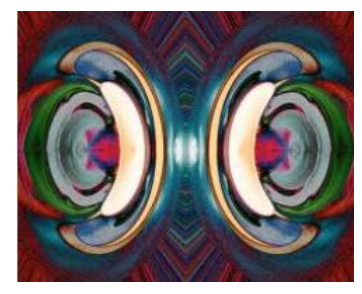

Figura 56:

A seguir são propostas algumas atividades com o propósito de desenvolver a percepção e abstração dos conceitos e propriedades de simetrias de Reflexão e Rotação. Inicialmente serão desenvolvidas atividades com auxílio de dobraduras e desenhos em papel quadriculado dando ênfase nas coordenadas cartesianas. Usaremos também conjunto de espelhos para uma melhor compreensão de eixos de simetrias e movimentos de rotação, além de recorrermos a pavimentação do plano.

\section{$7 \quad$ Atividades}

\section{Atividade 1}

Dobre uma folha ao meio, faça recortes formando um desenho, em seguida desdobre e responda:

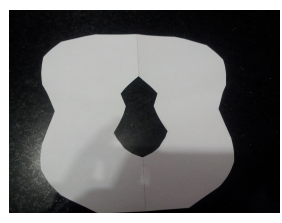

Figura 57: 
O que você observou no desenho obtido em ambos os lados em relação a dobra?

\section{Atividade 2}

Verifique se as figuras possuem eixos de simetria.

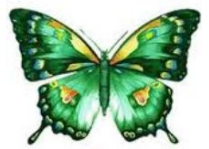

Figura 58:

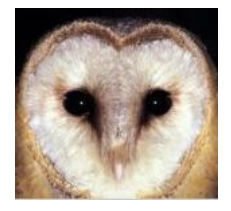

Figura 59:

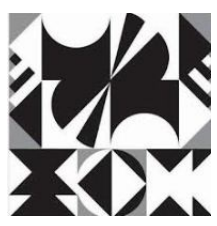

Figura 60:

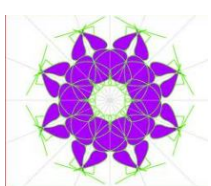

Figura 61:

\section{Atividade 3}

Dado um ponto A no plano cartesiano, localize o ponto B tal que seja a reflexão do ponto A em relação ao eixo Ox e responda:

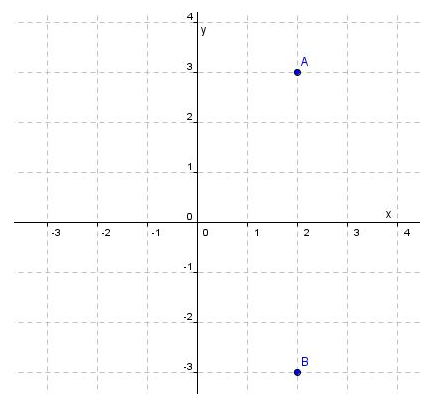

Figura 62:

Comparando as coordenadas do ponto $\mathrm{A}$ e do ponto $\mathrm{B}$, o que você pode concluir?

\section{Atividade 4}

Dado um ponto A no plano cartesiano, localize o ponto $\mathrm{C}$ tal que seja a reflexão do ponto $\mathrm{A}$ em relação ao eixo Oy e responda: 


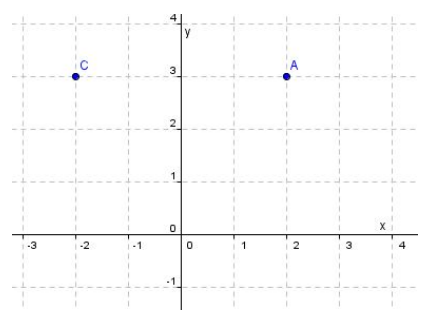

Figura 63:

Comparando as coordenadas do ponto $\mathrm{A}$ e do ponto $\mathrm{C}$, o que você pode concluir?

\section{Atividade 5}

Dado um triângulo (equilátero ou isósceles), o professor sugere que o aluno faça um desenho colorido e observe o que ocorre ao colocar o triângulo no livro de espelhos.

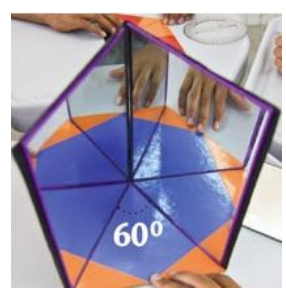

Figura 64:

Após a observação, o professor pode levantar questionamentos como:

Qual forma geométrica foi obtida?

Qual o ângulo formado entre os espelhos?

Existe algum tipo de simetria na figura formada pelo livro de espelhos?

\section{Atividade 6}

Através de vários recortes de uma mesma figura, o professor propõe aos alunos que façam os encaixes ou realizem os giros necessários para pavimentação do plano, como exemplificado na figura a seguir. 


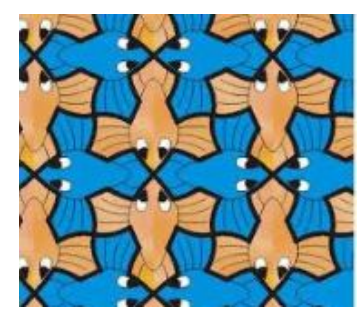

Figura 65:

\section{Atividade 7}

De quantos graus o floco de neve da figura 66 foi girado em torno de seu centro para obter a figura 67 ?

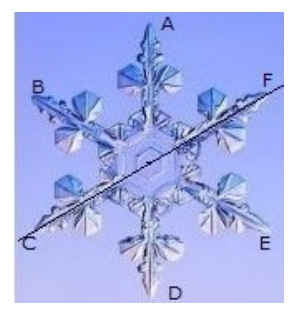

Figura 66:

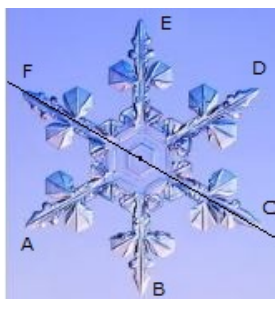

Figura 67:

\section{Atividade 8}

Nomeando os pontos do floco de neve, represente todos os giros, distintos, possíveis. Quantos giros diferentes podemos dar no floco de neve da figura sem mudá-lo aparentemente?

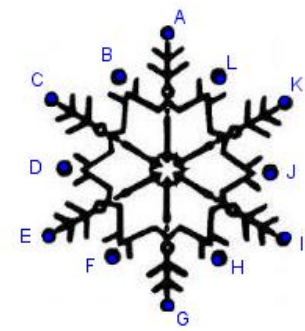

Figura 68: 


\section{Atividade 9}

Quantos eixos de simetria possui o floco de neve da figura? Represente todas as reflexões, distintas, possíveis.

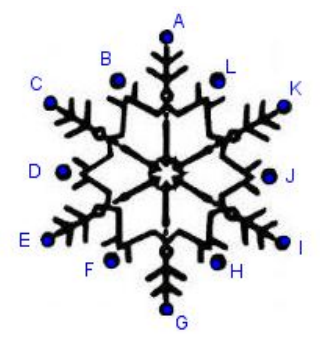

Figura 69:

\section{Atividade 10}

Das atividades 8 e 9 é possível calcular quantas simetrias, incluindo a identidade, o floco de neve da figura possui?

\section{Atividade 11}

O conjunto das simetrias de uma figura compõe o grupo de simetrias dessa figura. Assim dado um losango, não quadrado, represente todas as simetrias possíveis.

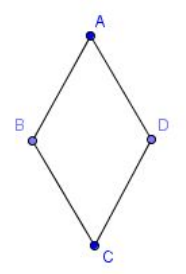

Figura 70:

\section{Atividade 12}

Dada a figura 


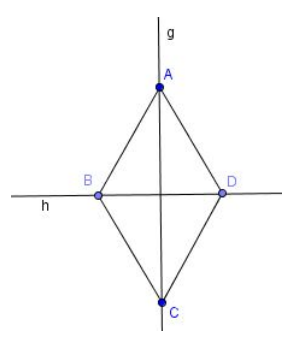

Figura 71:

Verifique qual simetria obtemos se aplicarmos :

a) a operação Identidade seguida da reflexão sobre o eixo de simetria h?

b) a operação rotação de $180^{\circ}$ em sentido anti-horário em torno do centro da figura seguida da Identidade?

c) a operação rotação de $180^{\circ}$ em sentido anti-horário em torno do centro da figura seguida da rotação de de $180^{\circ}$ em sentido horário?

d) a operação reflexão sobre o eixo de simetria h seguida da reflexão sobre o eixo de simetria g?

\section{Atividade 13}

Dizemos que uma figura tem simetria de cardinalidade $n$ se o número de simetrias da figura for $n$. Assim, quais das figuras abaixo tem a mesma cardinalidade de simetria?

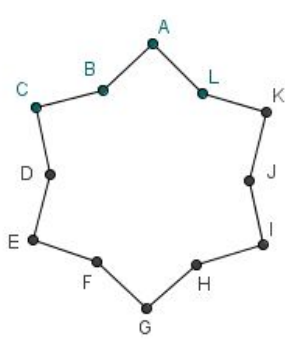

Figura 72:

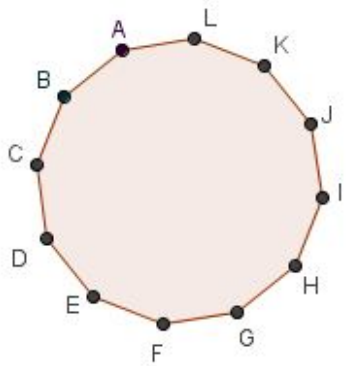

Figura 73:

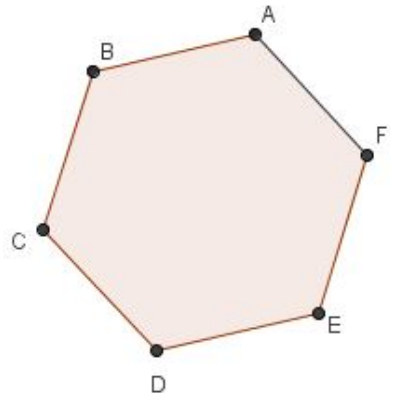

Figura 74:

\section{Atividade 14}

Considere o quadrado da figura 


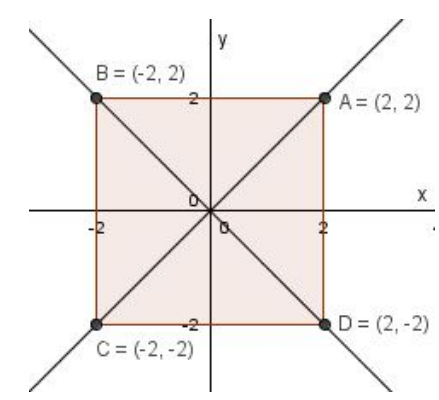

Figura 75:

Tomando $\theta=90^{\circ}$, calcule $x_{i}$ e $y_{i}$ tal que:

a) $x_{1}=x_{A} \cdot \cos \theta-y_{A} \cdot \operatorname{sen} \theta$ e $y_{1}=x_{A} \cdot \operatorname{sen} \theta+y_{A} \cdot \cos \theta$ onde, $x_{A}=2 \mathrm{e}$ $y_{A}=2$.

b) $x_{2}=x_{B} \cdot \cos \theta-y_{B} \cdot \operatorname{sen} \theta$ e $y_{2}=x_{B} \cdot \operatorname{sen} \theta+y_{B} \cdot \cos \theta$ onde, $x_{B}=-2 \mathrm{e}$ $y_{B}=2$.

c) $x_{3}=x_{C} \cdot \cos \theta-y_{C} \cdot \operatorname{sen} \theta$ e $y_{3}=x_{C} \cdot \operatorname{sen} \theta+y_{C} \cdot \cos \theta$ onde,$x_{C}=-2 \mathrm{e}$ $y_{C}=-2$.

d) $x_{4}=x_{D} \cdot \cos \theta-y_{D} \cdot \operatorname{sen} \theta$ e $y_{4}=x_{D} \cdot \operatorname{sen} \theta+y_{D} \cdot \cos \theta$ onde, $x_{D}=2 \mathrm{e}$ $y_{D}=2$.

Localize no plano cartesiano os pontos obtidos $\left(x_{1}, y_{1}\right),\left(x_{2}, y_{2}\right),\left(x_{3}, y_{3}\right)$ e $\left(x_{4}, y_{4}\right)$, trace segmentos unindo os pontos e a seguir o que você pode concluir da figura obtida em relação a figura dada? 


\section{Referências}

[1] A.Hefez, C.S.Fernadez: Introdução a Algebra Linear, Transformações Lineares e Matrizes, Coleção PROFMAT: SBM, 2012

[2] A. Azevedo, R.Piccinini: Introdução a Teoria dos Grupos, Grupos, Rio de Janeiro: IMPA, Setembro de 1969.

[3] M.L.T. Villela: Grupos, O conceito de grupo, Instituto de Matemática Universidade Federal Fluminense, Novembro de 2009.

[4] M. Spira: Grupos de Isometria no Plano, Isometrias, Agosto 1996

[5] M. M. P. Pinto: Grupos e Simetrias, Definição de grupos, Universidade Portucalense, Porto 2009

[6] R. L. Viana: Teoria dos Grupos, Grupos Discretos, Departamento de Física, Universidade Federal do Paraná, Curitiba 2010

[7] M. P. Florencio: Transformações no Plano e Grupos de Simetria, Isometrias no plano e grupos de simetria de polígonos regulares, UFSCar, São Carlos, Dezembro 2011.

[8] L. F. Lima: Grupos de Simetria I, Isomorfismos, UFSCar, São Carlos 2011.

[9] L. F. Lima: Grupos de Simetria II, Grupos Matriciais, UFSCar, São Carlos 2012

[10] pt.Wikipédia.org: Grupos de Simetria

[11] portaldoprofessor.mec.gov.br: Simetrias e dobraduras: Que monstro é esse?

[12] revistaescola.abril.com.br: Análise de simetrias com espelhos 